\title{
Circuit complexity as a novel probe of quantum entanglement: A study with black hole gas in arbitrary dimensions
}

\author{
Kiran Adhikari®, ${ }^{1}$ Sayantan Choudhury $\odot,{ }^{2,3, *}$ Satyaki Chowdhury, ${ }^{2,3}$ K. Shirish $\odot,{ }^{4}$ and Abinash Swain ${ }^{5}$ \\ ${ }^{1}$ Institute for Theoretical Particle Physics and Cosmology(TTK), RWTH Aachen University, \\ D-52056 Aachen, Germany \\ ${ }^{2}$ National Institute of Science Education and Research, Jatni, Bhubaneswar, Odisha-752050, India \\ ${ }^{3}$ Homi Bhabha National Institute, Training School Complex, Anushakti Nagar, Mumbai-400085, India \\ ${ }^{4}$ Visvesvaraya National Institute of Technology, Nagpur, Maharashtra 440010, India \\ ${ }^{5}$ Department of Physics, Indian Institute of Technology Gandhinagar, Palaj, Gandhinagar- 382355, India
}

(Received 3 June 2021; accepted 3 August 2021; published 2 September 2021)

\begin{abstract}
In this article, we investigate the quantum circuit complexity and entanglement entropy in the recently studied black hole gas framework using the two-mode squeezed states formalism written in arbitrary dimensional spatially flat cosmological Friedmann-Lemaitre-Robertson-Walker background space-time. We compute the various complexity measures and study the evolution of these complexities by following two different prescriptions viz the covariant matrix method and Nielsen's method. Independently, using the two-mode squeezed states formalism we also compute the Rényi and von-Neumann entanglement entropy, which show an inherent connection between the entanglement entropy and quantum circuit complexity. We study the behavior of the complexity measures and entanglement entropy separately for three different spatial dimensions and observe various significant different features in three spatial dimensions on the evolution of these quantities with respect to the scale factor. Furthermore, we also study the underlying behavior of the equilibrium temperature with two of the most essential quantities i.e., rate of change of complexity with scale factor and the entanglement entropy. We observe that irrespective of the spatial dimension, the equilibrium temperature depends quartically on entanglement entropy.
\end{abstract}

DOI: 10.1103/PhysRevD.104.065002

\section{INTRODUCTION}

Circuit complexity has become a helping hand to not only the high-energy physics community but also to the people from other branches as well [1-24]. This quantum information theory technique has been significantly used recently to probe many features which were previously difficult. Though this concept is a computation tool, its contribution in the field of physics of late is massive. It provides a way to probe physics behind the horizon of black holes through the use of the "Complexity = Volume" and "Complexity = Action" conjectures [25-29]. Since then it has been extensively used in quantum field theory and in studies involving AdS/CFT correspondence [30]. These holographic approaches connect a probe on the gravity side with a concept of quantum information theory.

\footnotetext{
* Corresponding author. sayantan.choudhury@niser.ac.in, sayanphysicsisi@gmail.com

Published by the American Physical Society under the terms of the Creative Commons Attribution 4.0 International license. Further distribution of this work must maintain attribution to the author(s) and the published article's title, journal citation, and DOI. Funded by SCOAP ${ }^{3}$.
}

In the recent past, along with the out of time ordered correlation functions [31-35], it has formed the web of quantum chaos. It has been found to reveal essential information like Lyapunov exponent, scrambling time etc., required to diagnose chaos in a system. Many interesting works have been done using this tool in wide areas of physics. It was studied for cosmological islands in [36], where the authors showed that entanglement entropy from circuit complexity via a famous relation proposed in [25] resembles the page curve in some particular regime where a universal relation between circuit complexity, out-of-timeordered-correlation (OTOC) and entanglement entropy can be written. It was used to study early Universe chaos within the framework of bouncing cosmology [37]. People have also computed circuit complexity in the context of supersymmetric quantum field theory [38]. The connection between entanglement and emergence of space-time has been an active area of study, where the entanglement entropy is the minimum cross-sectional area of an Einstein-Rosen Bridge (ERB). However, classically the ERB continues to grow for a very long time, whereas the dual thermodynamic system comes to a thermal equilibrium quickly. This led Susskind to introduce a new variable namely 'complexity' which could be responsible for the ERB growth [25]. 
Complexity of a quantum system is a real quantity and its growth rate has been conjectured to be proportional to the entropy of the black hole based on these observations. It was very recently shown in [39] that there exists some relationship between entangling power and circuit complexity. Most importantly, if the entanglement entropy grows linearly with time, the geometric circuit complexity also grows linearly.

In this paper, we will study the evolution of complexity with respect to the entanglement entropy of the black hole gas model [40]. An important feature of this model is that the total entropy of the black hole gas is directly proportional to the volume of the system instead of the area and the system behaves like a thermodynamic gas. We will compute the most common measures for determining the entanglement between the squeezed states of black hole gas, namely the von Neumann and Rényi entropy, which quantifies the amount of uncertainty linked to the density matrix. We will measure the entanglement entropy by constructing an effective thermal representation for the reduced single-mode state from the two-mode squeezed state which varies linearly with respect to squeezing parameter $r$. Then by using the scale factor predicted by the black hole gas model in the flat space-time metric as a dynamical variable we will study the evolution of complexity in three spatial dimensions and compare it with the entanglement entropy in terms of squeezed state parameters. One of the reasons we are interested in the black hole gas is that its equation of state describes a universe right after the big bang and before the start of inflation, if one wants to avoid such an equation of state governing radiations of very high densities then one needs to have inflation right from the Planck scale. The key highlights of this paper are as follows:

(a) The behavior of circuit complexity calculated from two different approaches viz the covariance matrix method and Nielsen's wave function method has been studied with respect to scale factor for the black hole gas model. We observe interesting behaviors for different spatial dimensions.

(b) The behavior of the von-Neumann entropy and the Rényi entropy has been studied with respect to the scale factor for different spatial dimensions. We observe similar features for $d=1,2$, whereas for $d=3$ we observe slightly different behavior.

(c) The behavior of $d C / d a$ with respect to von-Neumann and Rényi entropy has been studied for different spatial dimensions. It is shown that by no means is it a linear function.

(d) The behavior of the equilibrium temperature for the blackhole gas model is identical compared to entropy for different spatial dimensions whereas it seems dependent on spatial dimension when compared to $d C / d a$.

The organization of the paper is as follows: We begin by providing a review of the black hole gas given by Samir
Mathur in [40] in Sec. II. Solving the Friedmann equation for blackhole gas, we relate the scale factor $a(t)$ with spatial dimension. We also examine black hole gas perturbation theory in $(d+1)$ dimensions and we investigate the squeezed state formalism by perturbing the black hole gas geometry in Friedmann-Lemaître-Robertson-Walker (FLRW) spatially flat spacetime. In Sec. III we review the circuit complexity and we discuss the geometric framework of circuit complexity developed by Nielsen and collaborators. In Sec. IV after introducing the notion of squeezed states, we calculate its circuit complexity using two approaches: Complexity using the covariance matrix and Nielsen's method of wave functions. In Sec. V we compute the entanglement entropy of two-mode squeezed states. We also compute Rényi-entropy, von-Neumann entropy and Rényi-2 entropy. We find entanglement entropy grows linearly with increasing squeezing parameters for the short time period. Then, we compare entanglement entropy with circuit complexity obtained in Sec. I. In Sec. VI we numerically study the behavior of the complexity measures and entanglement entropy separately for three spatial dimensions $(d=1,2$, and 3$)$. In Sec. VII we conclude with some discussions.

\section{A SHORT NOTE ON BLACK HOLE GAS}

In this section, we review a model proposed in Ref. [40], where the author has studied the state of a system moving towards maximal entropy $S$. Note that this system is unlike the inflationary model where we have a low entropy state after the inflation because the positive energy of the matter content is compensated by the gravitational potential. Here, we provide a quick derivation of the equation of state that describes the preinflationary Universe. We will consider a configuration where we find entropy $S(E, V)$ of a system in a toroidal box of volume $V$ in the limit $E \rightarrow \infty$. At low enough energies the matter phase corresponds to radiation whose entropy as a function of $E$ and $V$ in dimension $d$ can be given by $S \approx V \rho^{\frac{d}{d+1}}$. By putting more energy into the box one can look for a configuration where the system turns into a black hole of radius $R$ whose entropy is given by

$$
S_{\text {hole }}=\frac{A}{G} .
$$

One might suspect that for a given box of radius $R$, Eq. (1) describes the state with maximum entropy for energy $E=E_{b h}$, since throwing more energy into the black hole will only result in increasing the Hubble expansion. However, if we let go the constraint that the energy inside the box shouldn't be greater than the mass of the black hole inside the box then one could arrive at a configuration where the entropy of the system is greater than (1). This could be achieved by putting $N$ number of black holes, each of radius $R$ in a lattice instead of a single box of volume $V$. The number of black holes in such a configuration is given as 


$$
N_{\text {hole }}=\left(\frac{V}{R^{d}}\right)
$$

Therefore the total entropy of the system is

$$
S=N_{\text {hole }} S_{\text {hole }}=\left(\frac{V}{R^{d}}\right)\left(\frac{R^{d-1}}{G}\right)=\frac{V}{R G} .
$$

We notice that (3) is in contrast with (1) where the entropy is directly proportional to the volume rather than the area of the horizon. The energy leading to such a state is undoubtedly greater than $E_{b h}$ and could be expressed as follows:

$$
E=N_{\text {hole }} E_{\text {hole }}=\left(\frac{V}{R^{d}}\right)\left(\frac{R^{d-2}}{G}\right)=\frac{V}{R^{2} G} .
$$

Substituting the value of $R$ from (4) to (3) we get

$$
S=\left(\frac{V}{E G}\right)^{-\frac{1}{2}}
$$

and for $\rho=E / V$ we get,

$$
S=K \sqrt{\frac{\rho}{G}} V .
$$

This contrast in the definition of entropy is subject to the constraint that microstates cannot expand freely to a larger size unlike in asymptotically flat space where the entropy is given by the area law. Also the resulting lattice configuration with $E>E_{b h}$, having $N$ number of black holes, would not collapse to form one large black hole as the entropy corresponding to the lattice configuration is larger than a single black hole state in a box of volume $V$.

Now, from the first law of thermodynamics we can show

$$
\begin{gathered}
T=\left(\frac{\partial S}{\partial E}\right)^{-1}=\frac{2}{K} \sqrt{\frac{E G}{V}} \\
p=T\left(\frac{\partial S}{\partial V}\right)=\frac{E}{V}=\rho .
\end{gathered}
$$

Now from (8) we see that equation of state takes the form $\rho=w p$ with $w=1$. The solution for the black hole gas model can be obtained by starting with the FLRW flat metric in $(1+d)$ dimensions which is given by the following line element

$$
d s^{2}=-d t^{2}+a^{2}(t) d \vec{x}^{2} .
$$

Solving the Friedmann equation for black hole gas in the $(d+1)$-dimensional flat metric with scale factor $a(t)$ we get

$$
a(t)=a_{0} t^{1 / d} .
$$

The above FLRW flat metric can be written in terms of the conformal time scale by using the following conversion relation

$$
d \tau=\frac{d t}{a(t)} .
$$

Integrating both sides of the above equation we get the following relationship between the physical time $t$ and the conformal time $\tau$ in the arbitrary $(d+1)$-dimensional black hole gas,

$$
t= \begin{cases}\exp \left(a_{0} \tau\right) & d=1 \\ \left(\frac{a_{0}(1-d)}{d}\right)^{\frac{d}{d-1}} \tau^{\frac{d}{d-1}} & d>1 .\end{cases}
$$

This relationship is extremely useful for the present computation which helps us the directly translate the information in terms of desired conformal time from physical time.

In this conformal time coordinates, the flat FLRW line element gets transformed as

$$
d s^{2}=a^{2}(\tau)\left(-d \tau^{2}+d \vec{x}^{2}\right) .
$$

Hence, the above solution of the black hole gas scale factor can be written in terms of conformal time as follows:

$$
a(\tau)= \begin{cases}a_{0} \exp \left(a_{0} \tau\right) & d=1 \\ a_{0}\left(\frac{a_{0}(1-d)}{d}\right)^{\frac{1}{d-1}} \tau^{\frac{1}{d-1}} & d>1 .\end{cases}
$$

The corresponding Hubble parameter with respect to the conformal time scale can also be written as

$$
\mathcal{H}(\tau)= \begin{cases}a_{0} & d=1 \\ \frac{1}{d-1} \frac{1}{\tau} & d>1\end{cases}
$$

Now we will describe an equivalent scenario inside a black hole gas where one can obtain the above mentioned scale factor and Hubble parameter in any arbitrary $(d+1)$ dimensions. In this scenario we embed a scalar field within the framework of Einstein gravity having the previously mentioned spatially flat FLRW space-time, where the solution of the scale factor is exactly same as mentioned earlier. In this framework the representative action of the scenario is described as

$$
S=\frac{1}{2} \int d^{d+1} x \sqrt{-g}\left[R-(\partial \phi)^{2}-V(\phi)\right],
$$

where we have fixed the Planck mass $M_{p}=1$ for the present computation. The first term in the action represents 
the usual Einstein Hilbert term, the second term represents the kinetic term of the embedded scalar field. and the last term represents the potential function in a black hole gas $V(\phi)$ in any arbitrary $(d+1)$ dimensions. We have found the following two possibilities of the potential functions are allowed in the present context which can finally give rise to same same scale factor which we mentioned earlier,

$$
V(\phi)= \begin{cases}\frac{3}{d^{2}} \exp (-\sqrt{2 d} \phi) & \text { Choice I } \\ \frac{3}{d^{2}} \exp (\sqrt{2 d} \phi) & \text { Choice II. }\end{cases}
$$

Here it is important to note that, since we have embedded the scalar field in the homogeneous and isotropic spatially flat FLRW background it turns out to be the field is only a function of the time coordinate.

Further solving the Klein Gordon field equation in $d+1$ dimension spatially flat FLRW background the dynamical solution of the homogeneous and isotropic background scalar field $\phi$ in terms of the conformal time coordinate can be expressed as:

$$
\phi(\tau)=\mp \frac{\sqrt{2}}{d-1} \ln \left(\frac{a_{0}(d-1)}{d} \tau\right) .
$$

This solution actually representing the dynamics of the field inside the black hole gas in $(d+1)$-dimensional spatially flat FLRW background.

Now using this solution one can find out the following conformal time dependence of the potential function in the context of black hole gas

$$
V(\tau)= \begin{cases}3 \exp \left(-2 a_{0} \tau\right) & d=1 \\ \frac{3}{d^{2}}\left(\frac{a_{0}(1-d)}{d} \tau\right)^{-\frac{2 d}{d-1}} & d>1 .\end{cases}
$$

In Figs. 1 and 2, we plotted the two choices of fields with respect to the conformal time. In choice I, we see that for

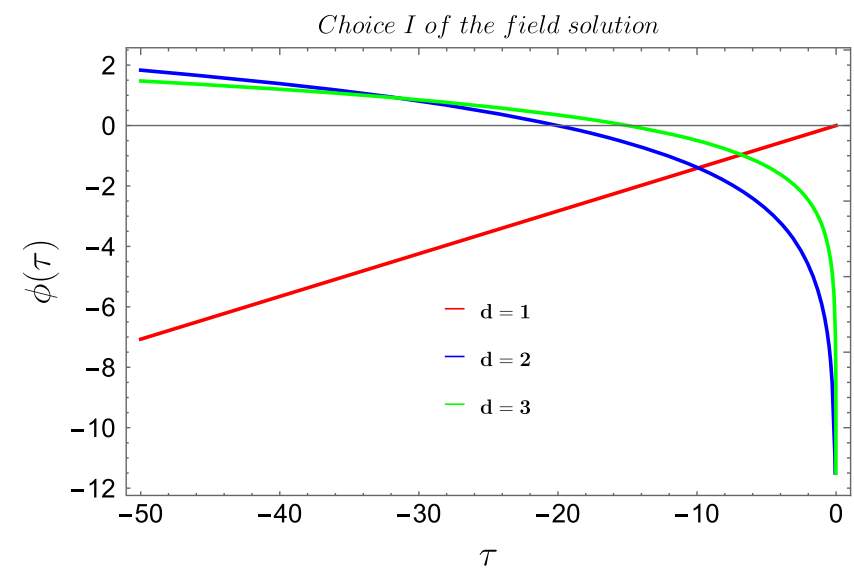

FIG. 1. Behavior of the first choice of the field solution with respect to conformal time for different spatial dimensions.

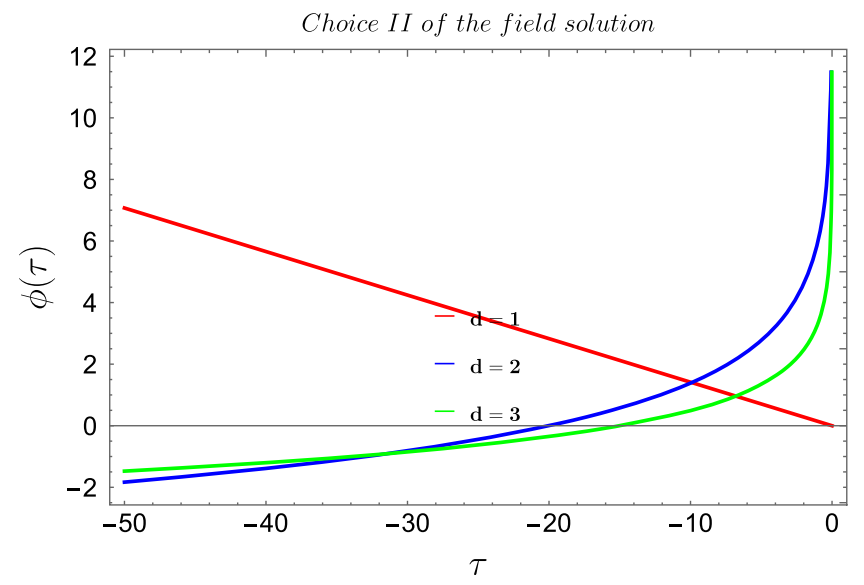

FIG. 2. Behavior of the second choice of the field solution with respect to conformal time for different spatial dimensions.

$d=1$ the field keeps on increasing monotonically and for higher dimensions i.e., $d=2,3$ the field value decreases slowly initially with respect to conformal time and then rapidly as we increase the value of conformal time. The exact opposite behavior can be seen for choice II. For $d=1$ the field value keeps on decreasing monotonically, where as for $d=2,3$ the field value grows steadily and then at a faster rate with conformal time.

In Figs. 3 and 4, we plotted potential for the two choices against the field. As can be seen from Eq. (17), the potential function takes the form of exponentially decreasing and increasing function for first and second choices respectively.

In Fig. 5 we have drawn the potential function with respect to conformal time. We have taken the logarithm for the potential. It can be seen that for $d=1$, the potential decreases continuously in a straight line whereas for $d=2$, 3 the potential rises quickly after going through a slow rise period in the beginning.

In Fig. 6 we plotted the scale factor of the black hole gas model with respect to the conformal time scale. The plots

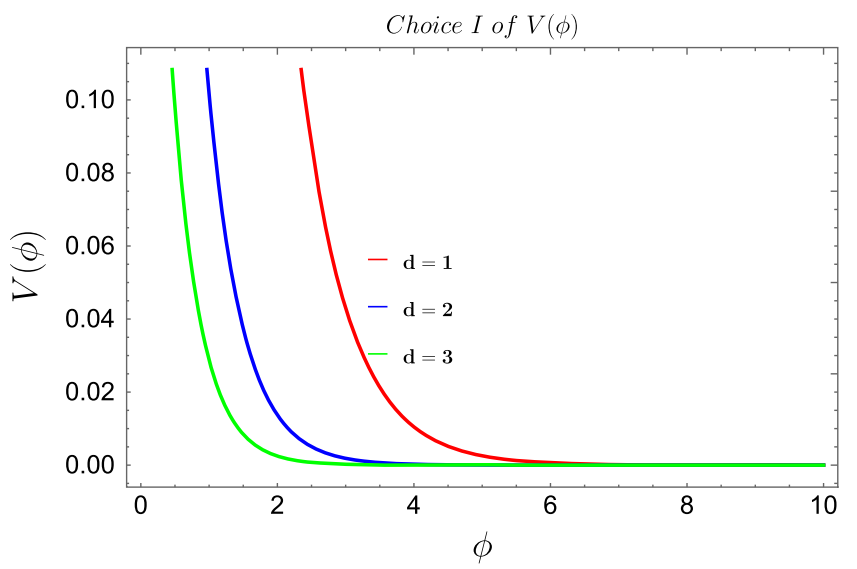

FIG. 3. Behavior of the first choice of the potential with respect to the field for different spatial dimensions. 


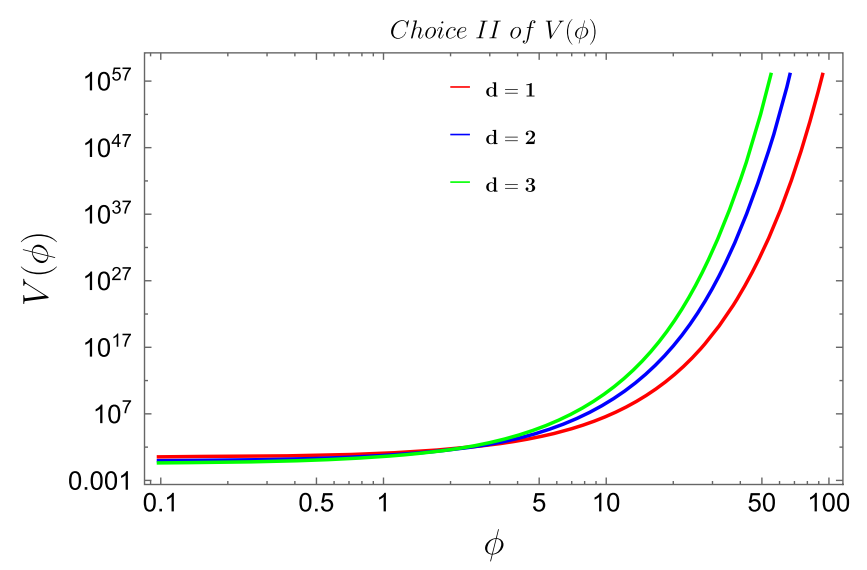

FIG. 4. Behavior of the second choice of the potential with respect to the field for different spatial dimensions.

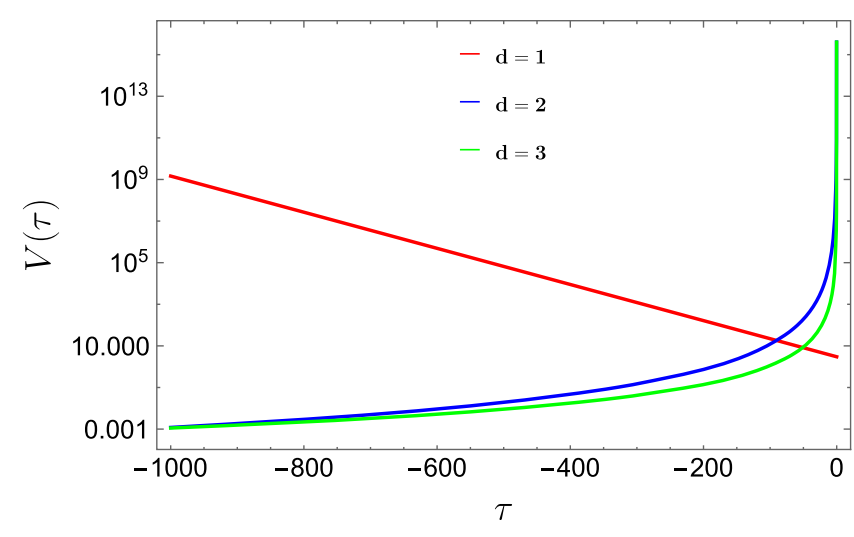

FIG. 5. Behavior of the potential with respect to the conformal time. Here we have taken the logarithm along the vertical axis.

have been done by fixing the value of the constant $a_{0}$ to 1 . We observe a significant difference in the behavior of the scale factor for the spatial dimension $d=1$ and the higher spatial dimensions. The scale factor corresponding to the spatial dimension $d=1$ shows increasing behavior in the

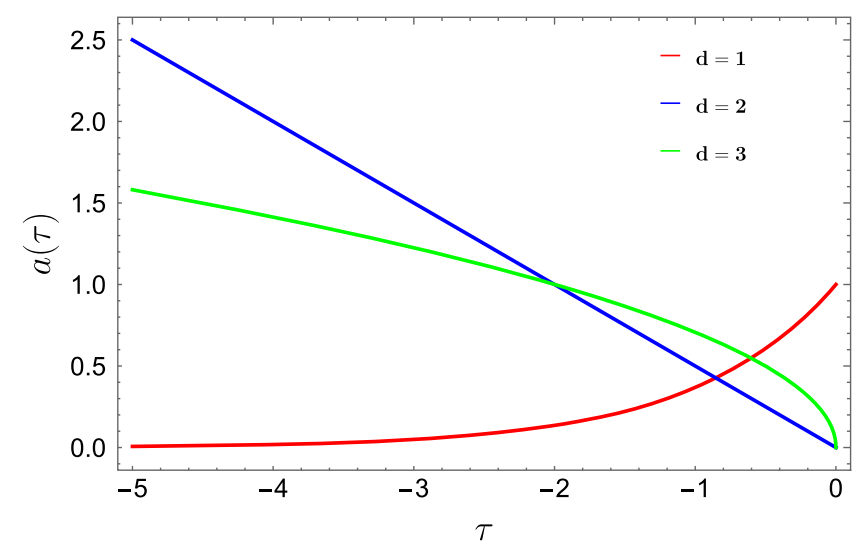

FIG. 6. Behavior of the scale factor $a(\tau)$ with respect to the conformal time for different spatial dimensions.

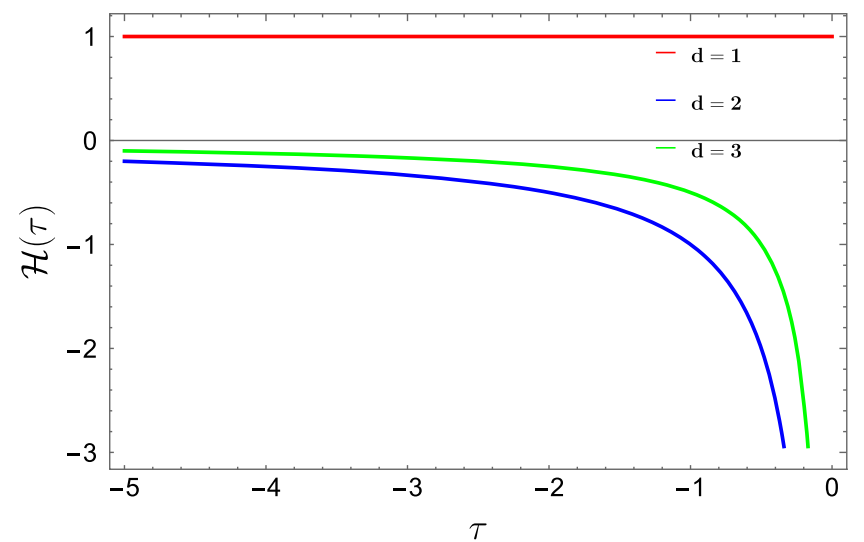

FIG. 7. Behavior of the Hubble parameter $\mathcal{H}(\tau)$ with respect to the conformal time for different spatial dimensions.

late time scales. The scale factor for higher spatial dimensions shows a decreasing behavior. The decrease is linear for the spatial dimension $d=2$ whereas it is nonlinear for spatial dimension $d=3$.

In Fig. 7 we plotted the behavior of the Hubble parameter $\left(\mathcal{H}(\tau)=\frac{a^{\prime}(\tau)}{a(\tau)}\right)$ with respect to the conformal time scale. It can be seen that the Hubble parameter is just a positive constant for the spatial dimension $d=1$, whereas it exhibits a decreasing behavior approaching negative infinity at late conformal time scales (near $\tau=0$ ) for the other spatial dimensions. Moreover, the value taken by the Hubble parameter for the higher spatial dimensions is always negative.

One could also arrive at (6) by using the $T$ and $S$ duality symmetries of string theory; this seems interesting because it does not require the state of black hole gas to be a state near the big bang since microstates of stringy theory could also describe the microstates of black holes.

\section{A. Black hole gas perturbation theory in $(d+1)$ dimensions}

In this section we will study squeezed state formalism within the framework of black hole gas theory for FLRW spatially flat background. In this context one needs to consider the following perturbation in the scalar field:

$$
\phi(\vec{x}, \tau)=\phi(\tau)+\delta \phi(\vec{x}, \tau),
$$

and to express the whole dynamics in terms of a gauge invariant description through a variable

$$
\zeta(\vec{x}, \tau)=-\frac{\mathcal{H}(\tau)}{\left(\frac{d \phi(\tau)}{d \tau}\right)} \delta \phi(\vec{x}, t) .
$$

At the level of first-order perturbation theory in a spatially flat FLRW background metric, we fix the following gauge constraints: 


$$
\begin{aligned}
\delta \phi(\vec{x}, \tau) & =0, \\
g_{i j}(\vec{x}, \tau) & =a^{2}(\tau)\left[(1+2 \zeta(\vec{x}, \tau)) \delta_{i j}+h_{i j}(\vec{x}, \tau)\right], \\
\partial_{i} h_{i j}(\vec{x}, \tau) & =0=h_{i}^{i}(\vec{x}, \tau),
\end{aligned}
$$

which fix the space-time reparametrization. In this gauge the spatial curvature of constant hypersurface vanishes, which implies the curvature perturbation variable is conserved outside the horizon. Applying the ADM formalism one can further compute the second-order perturbed action for scalar modes. The action, after gauge fixing, can then be expressed by the following:

$$
\begin{aligned}
\delta^{(2)} S= & \frac{1}{2} \int d \tau d^{d} \vec{x} \frac{a^{d-1}(\tau)}{\mathcal{H}^{2}}\left(\frac{d \phi(\tau)}{d \tau}\right)^{2} \\
& \times\left[\left(\partial_{\tau} \zeta(\vec{x}, \tau)\right)^{2}-\left(\partial_{i} \zeta(\vec{x}, \tau)\right)^{2}\right] .
\end{aligned}
$$

Now introducing the Mukhanov variable, defined as $v(\vec{x}, \tau)=z(\tau) \zeta(\vec{x}, \tau) \quad$ where, $\quad z(\tau)=a^{\frac{d-1}{2}}(\tau) \sqrt{2 \epsilon(\tau)}$; the second-order perturbed action can be rewritten as

$$
\begin{aligned}
\delta^{(2)} S= & \int d \tau d^{d} \vec{x}\left[v^{\prime 2}(\vec{x}, \tau)-\left(\partial_{i} v(\vec{x}, \tau)\right)^{2}+\left(\frac{z^{\prime}(\tau)}{z(\tau)}\right)^{2}\right. \\
& \left.\times v^{2}(\vec{x}, \tau)-2\left(\frac{z^{\prime}(\tau)}{z(\tau)}\right) v^{\prime}(\vec{x}, \tau) v(\vec{x}, \tau)\right],
\end{aligned}
$$

where the quantity $\epsilon(\tau)$ is known as the conformal timedependent slow-roll parameter and is defined as

$$
\epsilon(\tau)=1-\frac{\mathcal{H}^{\prime}}{\mathcal{H}^{2}}=\frac{1}{\mathcal{H}^{2}}\left(\frac{d \phi(\tau)}{d \tau}\right)^{2} .
$$

For the black hole gas model, it is very easy to verify that the slow-roll parameter is equal to the dimension in which the black hole model is being considered i.e.,

$$
\epsilon(\tau)=d
$$

which is finally independent of conformal time coordinate $\tau$.

By implementing the following ansatz for the Fourier transformation

$$
v(\vec{x}, \tau):=\int \frac{d^{d} \vec{k}}{(2 \pi)^{d}} v_{\vec{k}}(\tau) \exp (-i \vec{k} \cdot \vec{x})
$$

the second-order perturbation for the scalar modes in Fourier space can be further recast as

$$
\begin{aligned}
\delta^{(2)} S= & \int d \tau d^{d} \vec{k}\left[\left|v_{\vec{k}}^{\prime}(\tau)\right|^{2}+\left(k^{2}+\left(\frac{z^{\prime}(\tau)}{z(\tau)}\right)^{2}\right)\right. \\
& \left.\times\left|v_{\vec{k}}(\tau)\right|^{2}-2\left(\frac{z^{\prime}(\tau)}{z(\tau)}\right) v_{\vec{k}}^{\prime}(\tau) v_{-\vec{k}}(\tau)\right] .
\end{aligned}
$$

Now, varying the above second order action, we get the following equation of motion

$$
v_{\vec{k}}^{\prime \prime}(\tau)+\omega^{2}(k, \tau) v_{\vec{k}}(\tau)=0 .
$$

The above equation is known as the Mukhanov-Sassaki equation with the frequency of the oscillator given by

$$
\omega^{2}(k, \tau):=k^{2}+m_{\mathrm{eff}}^{2}(\tau) .
$$

The conformal time dependent effective mass in the present computation is given by

$$
m_{\mathrm{eff}}^{2}(\tau)=-\frac{z^{\prime \prime}(\tau)}{z(\tau)}=\frac{1}{\tau^{2}}\left(\nu_{\mathrm{BHG}}^{2}(\tau)-\frac{1}{4}\right),
$$

with the conformal time dependent mass parameter for the black hole gas in arbitrary dimension up to leading-order terms is given by

$$
\begin{aligned}
\nu_{\mathrm{BHG}}(\tau) & \approx \frac{d}{2}-1+\left(1-\frac{1}{\epsilon(\tau)}\right) \frac{\mathcal{H}^{\prime \prime}}{\mathcal{H}^{2}} \\
& =\frac{d}{2}-1-\frac{2}{d}(1-d)^{3} \mathcal{H},
\end{aligned}
$$

where we can clearly observe that the slowly-varying conformal-time dependence is appearing from the third term where we have truncated the expansion. While computing this mass parameter we have used the following facts.

$$
\begin{gathered}
\frac{\mathcal{H}^{\prime}}{\mathcal{H}^{2}}=(1-d), \\
\frac{\mathcal{H}^{\prime \prime}}{\mathcal{H}^{2}}=2(1-d)^{2} \mathcal{H},
\end{gathered}
$$

where the explicit expression for the Hubble parameter in the conformal time coordinate is quoted in the previous section. Finally using this result we get the following simplified expression for the mass parameter for black hole gas.

$$
\nu_{\mathrm{BHG}}(\tau)= \begin{cases}\frac{d}{2}-1-\frac{2}{d}(1-d)^{3} a_{0} & d=1 \\ \frac{d}{2}-1+\frac{2}{d}(1-d)^{2} \frac{1}{\tau} . & d>1\end{cases}
$$

A general solution to the equation of motion is written as

$$
v_{\vec{k}}(\tau):=\sqrt{-\tau}\left[\mathcal{C}_{1} \mathcal{H}_{\nu_{\mathrm{BHG}}}^{(1)}(-k \tau)+\mathcal{C}_{2} \mathcal{H}_{\nu_{\mathrm{BHG}}}^{(2)}(-k \tau)\right],
$$

where $\mathcal{H}_{\nu_{\mathrm{BHG}}}^{(1)}(-k \tau)$ and $\mathcal{H}_{\nu_{\mathrm{BHG}}}^{(2)}(-k \tau)$ are Hankel functions of the first and second kind, respectively, with argument $-k \tau$ and order $\nu_{\mathrm{BHG}}$. The two integration constants can be fixed by the choice of various initial conditions. In this paper, we restrict our choice to only the Bunch-Davies vacuum case in which one fixes the initial conditions, $\mathcal{C}_{1}$ as 1 and $\mathcal{C}_{2}$ as 0 . 
However, it is generally difficult to work with these full solutions and one takes the asymptotic limits of the solutions which is given by

$$
\begin{aligned}
v_{\vec{k}}(\tau)= & \frac{2^{\nu_{\mathrm{BHG}}-\frac{d}{2}}(-k \tau)^{\frac{d}{2}-\nu_{\mathrm{BHG}}}}{\sqrt{2 k}}\left|\frac{\Gamma\left(\nu_{\mathrm{BHG}}\right)}{\Gamma\left(\frac{d}{2}\right)}\right|\left(1-\frac{i}{k \tau}\right) \\
& \times \exp \left(-i\left\{k \tau+\frac{\pi}{2}\left(\nu_{\mathrm{BHG}}-\frac{d}{2}\right)\right\}\right) f_{d}(k, \tau) .
\end{aligned}
$$

where the function $f_{d}(k, \tau)$ takes the following form

$$
\begin{aligned}
f_{d}(k, \tau)= & 2^{\frac{d-3}{2}}(-k \tau)^{\frac{3-d}{2}}\left|\frac{\Gamma(d / 2)}{\Gamma(3 / 2)}\right| \\
& \times \exp \left\{-i\left(\frac{\pi}{2}\left(\frac{d-3}{2}\right)\right)\right\} .
\end{aligned}
$$

The normalization with the factor $f_{d}(k, \tau)$ has been done in such a way that for spatial dimension $d=3$, it becomes $f_{3}(k, \tau)=1$. In Figs. 8 and 9, we plotted the behavior of the real and the imaginary part of the mode functions with respect to the conformal time. We observe an identical behavior for both the real and imaginary parts of the mode functions. It shows an oscillatory behavior with respect to the conformal time and the amplitude of oscillation decreases with the conformal time evolution. Also, it can be observed that the amplitude of the oscillation decreases with the increase in the number of spatial dimensions. From the mode functions, one calculates the conjugate momentum to the mode functions and thus constructs the classical Hamiltonian function. By promotion of the mode function and the conjugate momentum to quantum mechanical operators in the Heisenberg picture, one quantizes the Hamiltonian which is written as

$$
\begin{aligned}
\hat{H}(\tau) & =\frac{1}{2} \int d^{3} \vec{k}\left[\Omega_{\vec{k}}(\tau)\left(\hat{c}_{\vec{k}}^{\dagger} \hat{c}_{\vec{k}}+\hat{c}_{-\vec{k}}^{\dagger} \hat{c}_{-\vec{k}}+1\right)\right. \\
& \left.+i \lambda_{\vec{k}}(\tau)\left(e^{-2 i \phi_{\vec{k}}(\tau)} \hat{c}_{\vec{k}} \hat{c}_{-\vec{k}}-e^{2 i \phi_{\vec{k}}(\tau)} \hat{c}_{\vec{k}}^{\dagger} \hat{c}_{-\vec{k}}^{\dagger}\right)\right],
\end{aligned}
$$

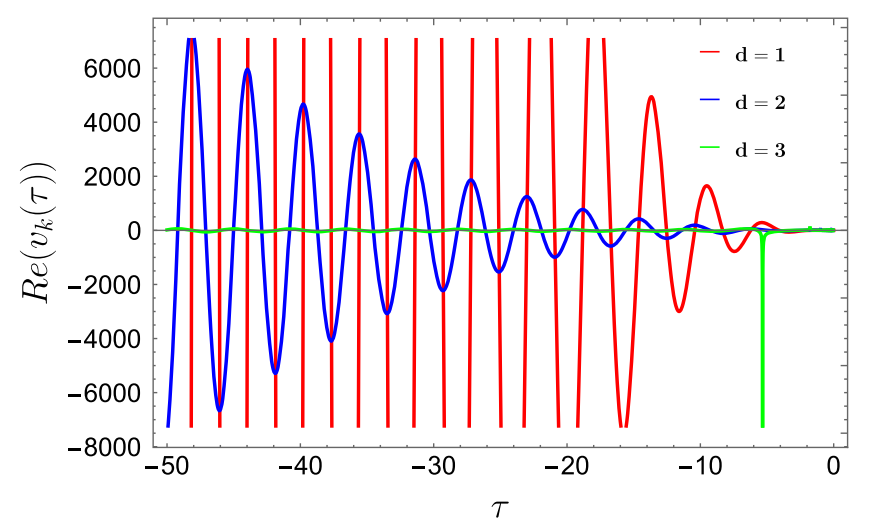

FIG. 8. Behavior of the real part of the mode solution $v_{k}$ with the scale factor.

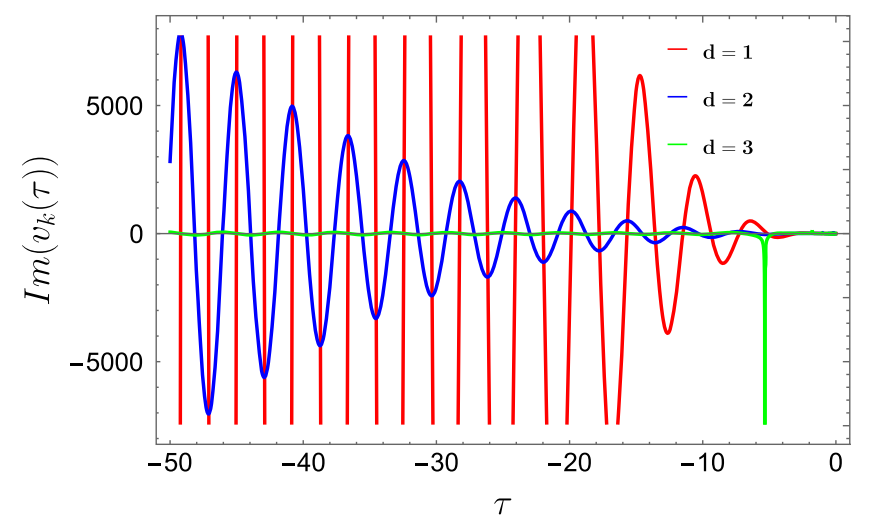

FIG. 9. Behavior of the imaginary part of the mode solution $v_{k}$ with the scale factor.

where, the origin of the creation and the annihilation operators can be understood, when one promotes the mode functions and its conjugate momentum to quantum mechanical operators. where the symbols $\Omega_{\mathbf{k}}(\tau)$ and $\lambda_{\mathbf{k}}(\tau)$ are defined by the following expressions

$$
\begin{gathered}
\Omega_{\vec{k}}(\tau):=\left\{\left|v_{\vec{k}}^{\prime}(\tau)\right|^{2}+\mu^{2}(k, \tau)\left|v_{\vec{k}}(\tau)\right|^{2}\right\}, \\
\lambda_{\vec{k}}(\tau):=\left(\frac{z^{\prime}(\tau)}{z(\tau)}\right),
\end{gathered}
$$

where the quantity $\mu^{2}(k, \tau)$ is given by

$$
\mu^{2}(k, \tau)=k^{2}-\lambda_{k}^{2}(\tau) .
$$

Imposing the initial condition at the horizon crossing $(k / \mathcal{H}=1)$, given by time scale $\left(\tau=\tau_{0}\right)$ which is given by the following conditions

$$
\text { Horizon crossing }= \begin{cases}\frac{k}{a_{0}}=1 & d=1 \\ k(d-1) \tau_{0}=1 & d>1,\end{cases}
$$

one can calculate the quantum operators at any arbitrary scale in the Heisenberg picture. Our next job is to determine the expression of the unitary operator in the context of cosmological primordial perturbations of the scalar modes where the concept of the squeezed state formalism plays a significant role. In this approach, the unitary operator is factorized as follows:

$$
\mathcal{U}\left(\tau, \tau_{0}\right)=\hat{\mathcal{S}}\left(r_{\vec{k}}\left(\tau, \tau_{0}\right), \phi_{\vec{k}}(\tau)\right) \hat{\mathcal{R}}\left(\theta_{\vec{k}}(\tau)\right),
$$

where $\mathcal{R}$ is the two-mode rotation operator which is defined as

$$
\hat{\mathcal{R}}\left(\theta_{\vec{k}}(\tau)\right)=\exp \left(-i \theta_{k}(\tau)\left(\hat{c}_{\vec{k}} \hat{c}_{\vec{k}}^{\dagger}+\hat{c}_{-\vec{k}}^{\dagger} \hat{c}_{-\vec{k}}\right)\right)
$$

and $\hat{\mathcal{S}}$ is the two-mode squeezing operator, defined as 


$$
\begin{aligned}
\hat{\mathcal{S}}\left(r_{\vec{k}}(\tau), \phi_{\vec{k}}(\tau)\right)= & \exp \left(r _ { \vec { k } } ( \tau ) \left[e^{-i \phi_{\vec{k}}(\tau)} \hat{c}_{\vec{k}} \hat{c}_{-\vec{k}}\right.\right. \\
& \left.\left.-e^{i \phi_{\vec{k}}(\tau)} \hat{c}_{-\vec{k}}^{\dagger} \hat{c}_{\vec{k}}^{\dagger}\right]\right) .
\end{aligned}
$$

Here the squeezing amplitude is represented by the timedependent parameter, $r_{\vec{k}}(\tau)$, and the squeezing angle or the phase is represented by the time-dependent parameter $\phi_{\vec{k}}(\tau)$. The two-mode rotation operator, $\hat{\mathcal{R}}$ produces an irrelevant phase contribution and is ignored. The ground state of the free part of the above Hamiltonian is taken as the initial quantum state, whereas the two-mode squeezedquantum vacuum state obtained by acting the squeezed operator on the initial vacuum is taken as the final target state. The time evolution of the conformal time-dependent quantum operators $\hat{\mathcal{R}}$ and $\hat{\mathcal{S}}$, described by the Schrödinger equation, gives the following set of differential equations for the squeezing parameters

$$
\begin{gathered}
\frac{d r_{\vec{k}}(\tau)}{d \tau}=-\lambda_{\vec{k}}(\tau) \cos \left(2 \phi_{\vec{k}}(\tau)\right) \\
\frac{d \phi_{\vec{k}}(\tau)}{d \tau}=\Omega_{\vec{k}}(\tau)+\lambda_{\vec{k}}(\tau) \operatorname{coth}\left(2 r_{\vec{k}}(\tau)\right) \sin \left(2 \phi_{\vec{k}}(\tau)\right)
\end{gathered}
$$

\section{A SHORT NOTE ON CIRCUIT COMPLEXITY}

One of the challenges in quantum information processing is to find out the efficient circuit for implementing a unitary operation $U$ which can be used to solve a computational problem like Search Algorithm or Shor's factoring [41-43]. In computer science, a similar term called complexity $[44,45]$ exists, which can be defined as the minimum number of computational gates required to implement a certain algorithm. If we extend this definition to quantum version as minimum number of quantum gates out of basic unitary gates [46] in order to implement a unitary operation $U$, we get quantum complexity $[47,48]$. Therefore, understanding the difficulty of implementing such unitary operation $U$, as a sequence of logical gates, is very helpful and at the same time challenging.

In Refs. [49-51], the authors introduced a geometric approach to compute quantum circuit complexity based on the idea that finding the optimal quantum circuit is equivalent to problems of computing geodesics in Riemannian geometry. Here, we define a Riemannian metric on the space of $n$-qubit operations, and the distance $d(I, U)$ between the identity and target unitary operation $U$ is equivalent to the number of quantum gates, which is then identified as the circuit complexity. It was shown that minimizing this distance $d(I, U)$ i.e., finding geodesic length, gives a good measure of complexity. Thus, one can employ well developed tools of Riemannian geometry such as the Levi-Civita connection, geodesics, curvature, etc. to analyze the quantum circuit complexity. It is important to note that, even though in Refs. [49-51] geometric techniques were introduced to study complexity, in Ref. [52], the authors have used previous techniques from the theory of symmetric spaces to study time-optimal control of quantum evolution.

Let $U$ be a transformation which transforms reference state $\left|\psi_{R}\right\rangle$ to the target state $\left|\psi_{T}\right\rangle$ via

$$
\left|\psi_{T}\right\rangle=U\left|\psi_{R}\right\rangle .
$$

The unitary transform $U$, in the language of quantum computation has an order of unitary gates $Q_{i}$ such that $U=$ $Q_{1} Q_{2} \ldots Q_{d}$ where $d$ is the depth of the circuit. We can also introduce the tolerance $\epsilon$ which tells us whether the transformation is successful

$$
||\left|\psi_{T}\right\rangle-U\left|\psi_{R}\right\rangle \|^{2} \leq \epsilon
$$

This makes sense because in any practical implications, it is difficult to represent the unitary transformation $U$ exactly as a combination of discrete unitary $Q_{i}$ operations.

Obviously, there exists infinite number of ways to achieve this target state $\left|\psi_{T}\right\rangle$ from the reference state. The circuit complexity is then the depth of the optimal circuit out of this infinite possibilities.

Motivated from the theory of Hamiltonian control problem, authors in Refs. [49-51] introduced a geometric approach to compute this circuit complexity which was later used to compute complexity in various quantum mechanical and quantum field theoretic models. Instead of directly counting discrete set of gates required for constructing $U$, Nielsen's approach is geometric. In this method, with a time-dependent Hamiltonian $H(t)$ one constructs unitary $U$ as

$$
\begin{aligned}
U & =\overleftarrow{\mathcal{P}} \exp \left[-i \int_{0}^{1} d \tau H(\tau)\right] \\
\text { where } H(\tau) & =\sum_{I} Y^{I}(\tau) \mathcal{O}_{I} .
\end{aligned}
$$

Here, the Hermitian operators $\mathcal{O}_{I}$ form the basis for time dependent Hamiltonian $H(\tau)$. The path-ordering operator $\mathcal{P}$ is another version of the time-ordering operator which indicates that the circuit, made out of noncommuting operators, is built from right to left. The right to left application of operators is a choice of convention. The control functions $Y^{I}(\tau)$ can be thought as particular gates added at a time $s$ represented by Eq. (51).

One can then construct paths in the space of unitaries as

$$
U=\overleftarrow{\mathcal{P}} \exp \left[-i \int_{0}^{\tau} d \tau^{\prime} H\left(\tau^{\prime}\right)\right]
$$

The most interesting case is when the trajectory satisfies the boundary conditions $U(\tau=0)=1$ and $U(\tau=1)=U$. These $\mathcal{O}_{I}$ and $Y^{I}(\tau)$ satisfy 


$$
Y^{I}(\tau) \mathcal{O}_{I}=\partial_{\tau} U(\tau) U^{-1}(\tau)
$$

Equation (53) is actually just a time-dependent Schrödinger equation in the form when one solves it via time-ordered exponentials.

As we discussed before, there are an infinite number of ways of achieving a unitary transformation $U$. However, not all processes are optimal. In order to find out the optimal transformation, a cost function $F(U, \vec{Y}(\tau))$ is defined. This cost function $F(U, \vec{Y}(\tau))$ is a local functional along the trajectory of the $U(\tau)$ and tangent vectors $\vec{Y}(\tau)$ ). Now, for each path the cost is defined as

$$
\mathcal{D}(U(t))=\int_{0}^{1} d t F(U(t), \dot{U}(t)) .
$$

Nielsen showed that, the variational geometric approach of minimizing this functional is basically finding the optimized quantum circuit. Standing on the physical grounds, the cost function $F$ should satisfy certain properties. Those are appended below.

(a) Continuity: $F \in C^{0}$ i.e., $F$ should be continuous. It is reasonable to assume continuity on physical grounds.

(b) Positivity: Based on the definition of cost function $F$, it is reasonable to expect

$$
F(U, v) \geq 0,
$$

where equality holds if and only if $v=0$. The equality condition also implies that the reference and target is basically same.

(c) Positive homogeneity: For any positive real number $\alpha$ and any vector $v$, we get $F(\alpha v)=\alpha F(v)$.

(d) Triangle Inequality: $F$ satisfies the triangle inequality,

$$
F\left(U, v+v^{\prime}\right) \leq F(U, v)+F\left(U, v^{\prime}\right),
$$

for all tangent vectors $v$ and $v^{\prime}$. The special case $F\left(U, v+v^{\prime}\right)=F(U, v)+F\left(U, v^{\prime}\right)$ is satisfied if and only if $v$ and $v^{\prime}$ are along the same ray coming out from the origin.

If one extends the continuity condition $F \in C^{0}$ with $F \in$ $C^{\infty}$ i.e., $F$ is smooth; then the manifold is known as Finsler Manifold. Nielsen's geometric approach of determining complexity is computing the geodesic in Finsler geometry, and the length of this geodesic gives the complexity. This definition of complexity is also called the geometric circuit complexity.

In literature, there are different choices of these cost functions $F(U, v)$. These choices depends on how one defines the complexity, and the elementary gate sets for their setup. Some of the simple examples are

$$
\begin{aligned}
& F_{1}(U, Y)=\sum_{I}\left|Y^{I}\right|, \\
& F_{p}(U, Y)=\sum_{I} p_{I}\left|Y^{I}\right|, \\
& F_{2}(U, Y)=\sqrt{\sum_{I}\left|Y^{I}\right|^{2}}, \\
& F_{q}(U, Y)=\sqrt{\sum_{I} q_{I}\left|Y^{I}\right|^{2}} .
\end{aligned}
$$

We can now give comments on various choices of these cost functions. $F_{1}$; the linear cost functional, measure is the nearest concept that is close to counting individual gates in the circuit. $F_{2}$; the quadratic cost functional, can be considered as the proper distance in the manifold. $F_{1 p}$ can be thought of as a cost function where penalty factors $p_{I}$ are used to favor certain directions over others. This becomes reasonable when one consider elementary gates coupled only to the neighboring qubits and discard those qubits which are nonlocal. Depending on the system, one can choose different cost functions to study the circuit complexity.

One can also introduce a general class of inhomogeneous and homogeneous family of functionals represented by

$$
\begin{aligned}
& F_{k}(U, Y)=\sum_{I}\left|Y^{I}\right|^{k}, \\
& F_{\frac{1}{k}}(U, Y)=\sum_{I}\left|Y^{I}\right|^{\frac{1}{k}},
\end{aligned}
$$

where $k \geq 1$ represents the degree of homogeneity. $F_{k}$ was introduced in the context of holography to match the results obtained from "Complexity = Action" and "Complexity = Volume" conjectures.

\section{CIRCUIT COMPLEXITY OF TWO MODE SQUEEZED STATES}

A simple but a very rich example of entangled multimode field states is the two-mode squeezed vacuum state. (More details about two-mode squeezed states can be found in [53].) As already defined in the previous section, the two-mode squeezing operator is given by

$$
\hat{S}_{\vec{k}}(\xi)=\exp \left(\xi^{*} \hat{c}_{\vec{k}} \hat{c}_{-k}-\xi \hat{c}_{\overrightarrow{-k}}^{\dagger} \hat{c}_{\vec{k}}^{\dagger}\right)
$$

where $\xi=r_{k} e^{i \phi_{k}}, r_{k}$ and $\phi_{k}$ are known as squeezing parameters and $0 \leq r_{k}<\infty$ and $0 \leq \phi_{k} \leq 2 \pi$. The twomode squeezed vacuum state, which will act as our target state is given by the action of two-mode squeezing operator $\hat{S}_{\vec{k}}(\xi)$ on the two-mode vacuum state (initial state), $|0\rangle_{\vec{k}}|0\rangle_{-k}=|0,0\rangle$. 


$$
\begin{aligned}
\left|\psi_{\mathrm{sq}}\right\rangle_{\vec{k},-\overrightarrow{ }} & =\hat{S}_{\vec{k}}(\xi)|0,0\rangle \\
& =\exp \left(\xi^{*} \hat{c}_{\vec{k}} \hat{c}_{\overrightarrow{-k}}-\xi \hat{c}_{-k}^{\dagger} \hat{c}_{\vec{k}}^{\dagger}\right)|0,0\rangle .
\end{aligned}
$$

In terms of the number of states, one can show that the state of two-mode squeezed states is given by

$\left.\left|\psi_{\mathrm{sq}}\right\rangle_{\vec{k}, \overrightarrow{-k}}=\frac{1}{\cosh r_{k}} \sum_{n=0}^{\infty}(-1)^{n} e^{i n \theta}\left(\tanh r_{k}\right)^{n}\left|n_{k}, n_{-k}\right\rangle\right)$.

We are now in the position to compute circuit complexity of the reference and target state of the two-mode squeezed states and compare it to the entanglement entropy. For this, we need to write our reference and target quantum states as Gaussian wave functions. The auxiliary position and momentum variables are

$$
\begin{aligned}
& \hat{q}_{\vec{k}}=\frac{1}{\sqrt{2 \Omega_{k}}}\left(\hat{c}_{\vec{k}}^{\dagger}+\hat{c}_{\vec{k}}\right), \\
& \hat{p}_{\vec{k}}=i \sqrt{\frac{\Omega_{k}}{2}}\left(\hat{c}_{\vec{k}}^{\dagger}-\hat{c}_{\vec{k}}\right),
\end{aligned}
$$

with, $\left[\hat{q}_{\vec{k}}, \hat{p}_{\vec{k}^{\prime}}\right]=i \delta^{3}\left(\vec{k}-\vec{k}^{\prime}\right)$. The reference state i.e., the two-mode vacuum state, in the position space can be expressed as a Gaussian wave function as follows:

$$
\begin{aligned}
\psi_{R}\left(q_{\vec{k}}, q_{-\vec{k}}\right) & =\left\langle q_{\vec{k}}, q_{-\vec{k}} \mid 0\right\rangle_{\vec{k},-\vec{k}} \\
& =\left(\frac{\Omega_{k}}{\pi}\right)^{\frac{1}{4}} \exp \left(-\frac{\Omega_{k}}{2}\left(q_{\vec{k}}^{2}+q_{-\vec{k}}^{2}\right)\right) .
\end{aligned}
$$

The target state, the two-mode squeezed state, in the position space has the wave function

$$
\begin{aligned}
\psi_{\mathrm{sq}}\left(q_{\vec{k}}, q_{-\vec{k}}\right) & =\left\langle q_{\vec{k}}, q_{-\vec{k}} \mid \psi_{\mathrm{sq}}\right\rangle_{\vec{k}} \\
& =\frac{e^{A\left(q_{\vec{k}}^{2}+q_{-\vec{k}}^{2}\right)-B q_{\vec{k}} q_{-\vec{k}}}}{\cosh r_{k} \sqrt{\pi} \sqrt{1-e^{-4 i \phi_{k} \tanh ^{2} r_{k}}}},
\end{aligned}
$$

where, $A$ and $B$ are the coefficients and are functions of squeezing parameters $r_{k}$ and $\phi_{k}$

$$
\begin{aligned}
& A=\frac{\Omega_{k}}{2} \frac{e^{-4 i \phi_{k} \tanh ^{2} r_{k}+1}}{e^{-4 i \phi_{k}} \tanh ^{2} r_{k}-1}, \\
& B=2 \Omega_{k} \frac{e^{-2 i \phi_{k}} \tanh r_{k}}{e^{-4 i \phi_{k}} \tanh ^{2} r_{k}-1} .
\end{aligned}
$$

It is helpful to define three other terms for simplifying the complexity calculation

$$
\Sigma_{\vec{k}}=-2 A+B, \quad \Sigma_{-\vec{k}}=-2 A-B, \quad \omega_{\vec{k}}=\omega_{-\vec{k}}=\frac{\Omega_{k}}{2} .
$$

Three methods of computing complexity were discussed in [54]. For our case, two methods i.e., computing complexity via covariance matrix method and Nielsen's method are relevant. As we discussed before in Eqs. (57) and (58), complexity depends on the choice of cost functions. Let $C_{1}$ be the circuit complexity corresponding to linear cost functional $F_{1}, C_{2}$ to quadratic cost functional $F_{2}$, and $C_{k}$ to k family of functionals $F_{k}$.

\section{A. Complexity via the covariance matrix method}

This method is interesting because complexity from the covariance matrix method is independent of the squeezing angle $\phi_{k}$. We will see later that the entanglement entropy obtained is also independent of the squeezing angle $\phi_{k}$, so the comparison between circuit complexity and entanglement entropy is more visible in this approach. Since our reference and target states (64) and (65) are in Gaussian form, we can express it as covariance matrix. The covariance matrix for the reference state is given as

$$
G_{k}^{s=0}=\left[\begin{array}{cccc}
\frac{1}{\Omega_{k}} & 0 & 0 & 0 \\
0 & \Omega_{k} & 0 & 0 \\
0 & 0 & \frac{1}{\Omega_{k}} & 0 \\
0 & 0 & 0 & \Omega_{k}
\end{array}\right] .
$$

The covariance matrix for the target state is given as

$$
G_{k}^{s=1}=\left[\begin{array}{cccc}
\frac{1}{\operatorname{Re}\left(\Sigma_{\vec{k}}\right)} & -\frac{\operatorname{Im}\left(\Sigma_{\vec{k}}\right)}{\operatorname{Re}\left(\Sigma_{\vec{k}}\right)} & 0 & 0 \\
-\frac{\operatorname{Im}\left(\Sigma_{\vec{k}}\right)}{\operatorname{Re}\left(\Sigma_{\vec{k}}\right)} & \frac{\left|\Sigma_{\vec{k}}\right|^{2}}{\operatorname{Re}\left(\Sigma_{\vec{k}}\right)} & 0 & 0 \\
0 & 0 & \frac{1}{\operatorname{Re}\left(\Sigma_{-\vec{k}}\right)} & -\frac{\operatorname{Im}\left(\Sigma_{-\vec{k}}\right)}{\operatorname{Re}\left(\Sigma_{-\vec{k}}\right)} \\
0 & 0 & -\frac{\operatorname{Im}\left(\Sigma_{-\vec{k}}\right)}{\operatorname{Re}\left(-\Sigma_{\vec{k}}\right)} & \frac{\left|\Sigma_{-\vec{k}}\right|^{2}}{\operatorname{Re}\left(\Sigma_{-\vec{k}}\right)}
\end{array}\right],
$$

where $\Sigma_{\vec{k}}$ and $\Sigma_{-\vec{k}}$ are defined in (67). The covariance matrix basically carries the same information as the wave function. In the context of the covariance matrix approach, circuit complexity quantifies the number of gates to take the covariance matrix of the reference state to the covariance matrix of the target state. We will factorize the covariance matrix $G$ into two $2 \times 2$ matrices. This gives us the benefit that we can compute complexity for each block and sum over all $\Omega_{k}$ to give the total complexity. The two symmetric blocks for the reference states are

$$
G_{k=0}^{s=0}=\left[\begin{array}{cc}
\frac{1}{\Omega_{k}} & 0 \\
0 & \Omega_{k}
\end{array}\right], \quad G_{k=1}^{s=0}=\left[\begin{array}{cc}
\frac{1}{\Omega_{k}} & 0 \\
0 & \Omega_{k}
\end{array}\right],
$$

while, the two symmetric blocks for the target states are 


$$
\begin{gathered}
G_{k=0}^{s=1}=\left[\begin{array}{cc}
\frac{1}{\operatorname{Re}\left(\Sigma_{\vec{k}}\right)} & -\frac{\operatorname{Im}\left(\Sigma_{\vec{k}}\right)}{\operatorname{Re}\left(\Sigma_{\vec{k}}\right)} \\
-\frac{\operatorname{Im}\left(\Sigma_{\vec{k}}\right)}{\operatorname{Re}\left(\Sigma_{\vec{k}}\right)} & \frac{\left|\Sigma_{\vec{k}}\right|^{2}}{\operatorname{Re}\left(\Sigma_{\vec{k}}\right)}
\end{array}\right], \\
G_{k=1}^{s=1}=\left[\begin{array}{cc}
\frac{1}{\operatorname{Re}\left(\Sigma_{-\vec{k}}\right)} & -\frac{\operatorname{Im}\left(\Sigma_{-k}\right)}{\operatorname{Re}\left(\Sigma_{-k}\right)} \\
-\frac{\operatorname{Im}\left(\Sigma_{-\vec{k}}\right)}{\operatorname{Re}\left(\Sigma_{-k}\right)} & \frac{\left|\Sigma_{-k}\right|^{2}}{\operatorname{Re}\left(\Sigma_{-k}\right)}
\end{array}\right],
\end{gathered}
$$

The basis for each block is changed to make the calculation easier as follows:

$$
\tilde{G}^{s=1}=S G^{s=1} S^{T}, \quad \tilde{G}^{s=0}=S G^{s=0} S^{T},
$$

where $S$ is a specifically chosen matrix such that $\tilde{G}^{s=0}=\mathbb{1}$. In our case, the matrix $S$ is given by

$$
S=\left[\begin{array}{cc}
\sqrt{\Omega_{k}} & 0 \\
0 & \frac{1}{\sqrt{\Omega_{k}}}
\end{array}\right]
$$

This implies $\tilde{G}^{s=0}=\mathbb{1}$ and

$$
\tilde{G}^{s=1}=\left[\begin{array}{cc}
\frac{\Omega_{k}}{\operatorname{Re}\left(\Sigma_{\vec{k}}\right)} & -\frac{\operatorname{Im}\left(\Sigma_{\vec{k}}\right)}{\operatorname{Re}\left(\Sigma_{\vec{k}}\right)} \\
-\frac{\operatorname{Im}\left(\Sigma_{\vec{k}}\right)}{\operatorname{Re}\left(\Sigma_{\vec{k}}\right)} & \frac{\left|\Sigma_{\vec{k}}\right|^{2}}{\Omega_{k} \operatorname{Re}\left(\Sigma_{\vec{k}}\right)}
\end{array}\right] .
$$

We can assume that $k$ is real. In the language of the covariance matrix, the unitary transformation of wave functions can be expressed as

$$
\tilde{G}^{s}=\tilde{U}(\tau) \tilde{G}^{s=0} \tilde{U}(\tau)^{T} .
$$

The unitary transformation is then parametrized with gates satisfying $S L(2, R)$ algebra as

$$
\tilde{U}(\tau)=\left[\begin{array}{cc}
\cos (\mu(\tau)) \cosh (\rho(\tau))-\sin (\theta(\tau)) \sinh (\rho(\tau)) & -\sin (\mu(\tau)) \cosh (\rho(\tau))+\cos (\theta(\tau)) \sinh (\rho(\tau)) \\
\sin (\mu(\tau)) \cosh (\rho(\tau))+\cos (\theta(\tau)) \sinh (\rho(\tau)) & \cos (\mu(\tau)) \cosh (\rho(\tau))+\sin (\theta(\tau)) \sinh (\rho(\tau))
\end{array}\right],
$$

where, $\mu, \rho, \theta$ are the coordinates on the $S L(2, R)$ group. Now, we will set following boundary conditions:

$$
\begin{aligned}
\tilde{G}^{s=1} & =\tilde{U}(\tau=1) \tilde{G}^{s=0} \tilde{U}(\tau=1)^{T}, \\
\tilde{G}^{s=0} & =\tilde{U}(\tau=0) \tilde{G}^{s=0} \tilde{U}(\tau=0)^{T} .
\end{aligned}
$$

This boundary conditions applied with the parametrized unitary transformations gives

$$
\begin{aligned}
(\cosh (2 \rho(1)), \tan (\theta(1)+\mu(1))) & =\left(\frac{\Omega_{k}^{2}+\left|\Sigma_{k}\right|^{2}}{2 \Omega \operatorname{Re}\left(\Sigma_{k}\right)}, \frac{\Omega_{k}^{2}-\left|\Sigma_{k}\right|^{2}}{2 \Omega \operatorname{Im}\left(\Sigma_{k}\right)}\right), \\
(\rho(0), \theta(0)+\mu(0)) & =(0, c) .
\end{aligned}
$$

In order to make the calculation simpler we choose:

(1) $\mu(\tau=1)=\mu(\tau=0)=0$.

(2) $\theta(\tau=0)=\theta(\tau=1)=c=\tan ^{-1}\left(\frac{\Omega_{k}^{2}-\left|\Sigma_{k}\right|^{2}}{2 \Omega \operatorname{Im}\left(\Sigma_{k}\right)}\right)$.
Given these conditions the metric for $\tilde{U}$ becomes

$$
\begin{aligned}
d s^{2}= & d \rho^{2}+\cosh (2 \rho) \cosh ^{2} \rho d \mu^{2} \\
& +\cosh (2 \rho) \sinh ^{2} \rho d \theta^{2}-\sinh (2 \rho)^{2} d \mu d \theta .
\end{aligned}
$$

The simple geodesic is a straight line on this geometry which is given by $\rho(\tau)=\rho(1) \tau$. From, the boundary conditions (80), we get

$$
\rho_{k}(\tau=1)=\frac{1}{2} \cosh ^{-1}\left[\frac{\Omega_{k}^{2}+\left|\Sigma_{\vec{k}}\right|^{2}}{2 \Omega_{k} \operatorname{Re}\left(\Sigma_{\vec{k}}\right)}\right] .
$$

In order to get total circuit complexity, we have to sum over both values of $k$ i.e., $k$ and $-k$. Therefore, for linear and quadratic cost functions, $C_{1}$ and $C_{2}$, we will get

$$
\begin{aligned}
C_{1}\left(\Omega_{k}\right)= & \rho_{k}(\tau=1)+\rho_{-k}(\tau=1) \\
= & \frac{1}{2}\left[\cosh ^{-1}\left[\frac{\Omega_{k}^{2}+\left|\Sigma_{\vec{k}}\right|^{2}}{2 \Omega_{k} \operatorname{Re}\left(\Sigma_{\vec{k}}\right)}\right]\right. \\
& \left.+\cosh ^{-1}\left[\frac{\Omega_{-k}^{2}+\left|\Sigma_{-\vec{k}}\right|^{2}}{2 \Omega_{-k} \operatorname{Re}\left(\Sigma_{-\vec{k}}\right)}\right]\right],
\end{aligned}
$$


Using the explicit form of $\Sigma_{\vec{k}}, \Sigma_{-\vec{k}}$, the circuit complexity reduces to a simple form which is independent of the squeezing angle $\phi_{k}$

$$
\begin{gathered}
C_{1}\left(\Omega_{k}\right)=4 r_{k} \\
C_{2}\left(\Omega_{k}\right)=2 \sqrt{2} r_{k}
\end{gathered}
$$

These two cost functions are then related by $C_{1}\left(\Omega_{k}\right)=$ $\sqrt{2} C_{2}\left(\Omega_{k}\right)$. Naturally, for small squeezing parameters $r_{k} \rightarrow 0, C_{1} \approx 0$ and $C_{2} \approx 0$. This makes sense as for small squeezing parameters $r_{k}$, the reference and the target states are basically same.

\section{B. Complexity via Nielsen's wave-function method}

Unlike covariance matrix method, Nielsen's approach using wave functions gives the circuit complexity of twomode squeezed states that is sensitive to both squeezing parameters, $r_{k}$ and $\phi_{k}$. The general philosophy of computing circuit complexity is basically same as in the covariance approach. However, instead of representing the wave function as a covariance matrix, we will directly compute the complexity using the reference and target two-mode squeezed states, i.e., Eqs. (64) and (65) respectively. Then, we will be able to write the circuit complexity in terms of squeezing parameters $r_{k}$ and $\phi_{k}$.

The exponent of the target state i.e., two-mode squeezed states Eq. (65) can be diagonalized as

$$
\psi_{\mathrm{sq}}=\mathcal{N} \exp \left(-\frac{1}{2} \tilde{\mathcal{M}}^{a b} q_{a} q_{b}\right)
$$

where $\mathcal{N}$ is the normalization constant i.e., denominator in (65) and

$$
\tilde{\mathcal{M}}=\left[\begin{array}{cc}
-2 A+B & 0 \\
0 & -2 A-B
\end{array}\right]=\left[\begin{array}{cc}
\Sigma_{\vec{k}} & 0 \\
0 & \Sigma_{\overrightarrow{-k}}
\end{array}\right]
$$

The unsqueezed reference state is also a Guassian wave function represented by

$$
\begin{aligned}
\psi_{\mathrm{R}} & =\mathcal{N} \exp \left(-\frac{\Omega_{k}}{2}\left(q_{\vec{k}}^{2}+q_{-\vec{k}}^{2}\right)\right) \\
& =\mathcal{N} \exp \left(\frac{1}{2} \sum_{k,-k} \Omega_{k} \vec{k}^{2}\right) .
\end{aligned}
$$

Our two Gaussian wave functions have the form

$$
\psi^{\tau}=\mathcal{N} \exp \left(-\frac{1}{2}\left(v_{a} \cdot \mathcal{A}_{a b}^{\tau} \cdot v_{b}\right)\right)
$$

where $v=\left(q_{\vec{k}}, q_{-k}\right)$ and $\mathcal{A}^{\tau}$ is a $2 \times 2$ diagonal matrix. For the target state Eq. (87),

$$
\mathcal{A}^{\tau=1}=\mathcal{M}=\left[\begin{array}{cc}
\Sigma_{\vec{k}} & 0 \\
0 & \Sigma_{\overrightarrow{-k}}
\end{array}\right],
$$

while for our reference state Eq. (89), matrix $\mathcal{A}$ is $\mathcal{A}^{\tau=0}$. So,

$$
\mathcal{A}^{\tau=0}=\left[\begin{array}{cc}
\Omega_{k} & 0 \\
0 & \Omega_{-k}
\end{array}\right] .
$$

The unitary transformation Eq. (52) acts like,

$$
\mathcal{A}^{\tau}=\mathcal{U}(\tau) \cdot \mathcal{A}^{\tau=0} \cdot \mathcal{U}^{T}(\tau) .
$$

The boundary conditions are given by

$$
\begin{aligned}
& \mathcal{A}^{\tau=1}=\mathcal{U}(\tau=1) . \mathcal{A}^{\tau=0} \cdot \mathcal{U}^{T}(\tau=1), \\
& \mathcal{A}^{\tau=0}=\mathcal{U}(\tau=0) . \mathcal{A}^{\tau=0} \cdot \mathcal{U}^{T}(\tau=0) .
\end{aligned}
$$

$\mathcal{U}$ can be parametrized as in Eq. (51) such that at $\tau=1$, the required target state is achieved. Since, $\mathcal{A}^{\tau=1}$ and $\mathcal{A}^{\tau=0}$ can have complex elements, elementary gates are restricted to $G L(2, C)$ unitaries. Tangent vector components $Y^{I}$ in Eq. (53) are complex parameters while $\mathcal{O}_{I}$ are the generators. Equation (53) can also be expressed as

$$
Y^{I}=\operatorname{Tr}\left(\partial_{\tau} U(\tau) U^{-1}(\tau)\left(\mathcal{O}_{I}\right)^{T}\right)
$$

where, we note that

$$
\operatorname{Tr}\left(\mathcal{O}_{I} \cdot \mathcal{O}_{J}^{T}\right)=\delta^{I J}
$$

and $I, J=0,1,2,3$. The metric is then given by:

$$
d s^{2}=G_{I J} d Y^{I} d Y^{* J}
$$

For simplicity, we will choose penalty factors $G_{I J}=\delta^{I J}$ where we fix it to unity. The off-diagonal elements in $G L(2, C)$ can be set to zero as they increase the distance between states. The $U(\tau)$ will become

$$
U(\tau)=\exp \left(\sum_{i \in(k,-k)} \alpha^{i}(\tau) \mathcal{O}_{i}^{\text {diagonal }}\right),
$$

where, $\alpha^{i}(\tau)$ are complex parameters and $\mathcal{O}_{i}^{\text {diagonal }}$ are generators with identity at $i$ diagonal elements. The metric takes a simple form

$$
d s^{2}=\sum_{i \in(k,-k)}\left(d \alpha^{i, \operatorname{Re}}\right)^{2}+\left(d \alpha^{i, \operatorname{Im}}\right)^{2},
$$

where Re and Im indicates real and imaginary part of $\alpha_{k}$ respectively. The geodesic is again a straight line in the manifold given by 


$$
\alpha^{i, p}(\tau)=\alpha^{i, p}(\tau=1)+\alpha^{i, p}(\tau=0)
$$

for each $(i \in k,-k)$ and $(p=\operatorname{Re}$ and $\operatorname{Im})$. Given the boundary conditions, we will get,

$$
\begin{aligned}
\alpha^{i, \operatorname{Re}}(\tau=0) & =\alpha^{i, \operatorname{Im}}(\tau=0)=0, \\
\alpha^{i, \operatorname{Re}}(\tau=1) & =\frac{1}{2} \ln \left|\frac{\Sigma_{\vec{i}}}{\omega_{\vec{i}}}\right| \\
\alpha^{i, \operatorname{Im}}(\tau=1) & =\frac{1}{2} \tan ^{-1} \frac{\operatorname{Im}\left(\Sigma_{\vec{i}}\right)}{\operatorname{Re}\left(\Sigma_{\vec{i}}\right)},
\end{aligned}
$$

for each $(i \in k,-k)$. Now, the circuit complexity for linear $C_{1}\left(\Omega_{k}\right)$ and quadratic cost $C_{2}\left(\Omega_{k}\right)$ functions can be derived as follows:

$$
\begin{gathered}
C_{1}\left(\Omega_{k}\right)=\alpha^{k, \operatorname{Re}}(\tau=1)+\alpha^{-k, \operatorname{Re}}(\tau=1)+\alpha^{k, \operatorname{Im}}(\tau=1)+\alpha^{-k, \operatorname{Im}}(\tau=1) \\
=\frac{1}{2}\left(\ln \left|\frac{\Sigma_{\vec{k}}}{\omega_{\vec{k}}}\right|+\ln \left|\frac{\Sigma_{-\vec{k}}}{\omega_{-\vec{k}}}\right|+\tan ^{-1} \frac{\operatorname{Im}\left(\Sigma_{\vec{k}}\right)}{\operatorname{Re}\left(\Sigma_{\vec{k}}\right)}+\tan ^{-1} \frac{\operatorname{Im}\left(\Sigma_{-\vec{k}}\right)}{\operatorname{Re}\left(\Sigma_{-\vec{k}}\right)}\right), \\
C_{2}\left(\Omega_{k}\right)=\sqrt{\left(\alpha^{k, \operatorname{Re}}(\tau=1)\right)^{2}+\left(\alpha^{-k, \operatorname{Re}}(\tau=1)\right)^{2}+\left(\alpha^{k, \operatorname{Im}}(\tau=1)\right)^{2}+\left(\alpha^{-k, \operatorname{Im}}(\tau=1)\right)^{2}} \\
=\frac{1}{2} \sqrt{\left(\ln \left|\frac{\Sigma_{\vec{k}}}{\omega_{\vec{k}}}\right|\right)^{2}+\left(\ln \left|\frac{\Sigma_{-\vec{k}}}{\omega_{-\vec{k}}}\right|\right)^{2}+\left(\tan ^{-1} \frac{\operatorname{Im}\left(\Sigma_{\vec{k}}\right)}{\operatorname{Re}\left(\Sigma_{\vec{k}}\right)}\right)^{2}+\left(\tan ^{-1} \frac{\operatorname{Im}\left(\Sigma_{-\vec{k}}\right)}{\operatorname{Re}\left(\Sigma_{-\vec{k}}\right)}\right)^{2}} .
\end{gathered}
$$

Using explicit values of $\Sigma_{\vec{k}}, \Sigma_{-\vec{k}}, \omega_{\vec{k}}$, and $\omega_{-\vec{k}}$ from Eq. (67), we can get general circuit complexity form,

$$
\begin{gathered}
C_{1}\left(\Omega_{k}, \tau\right)=|\ln | \frac{1+\exp \left(-2 i \phi_{k}(\tau)\right) \tanh r_{k}(\tau)}{1-\exp \left(-2 i \phi_{k}(\tau)\right) \tanh r_{k}(\tau)}||+\left|\tanh ^{-1}\left(\sin \left(2 \phi_{k}(\tau)\right) \sinh \left(2 r_{k}(\tau)\right)\right)\right| \\
C_{2}\left(\Omega_{k}, \tau\right)=\frac{1}{\sqrt{2}} \sqrt{\left(\ln \left|\frac{1+\exp \left(-2 i \phi_{k}(\tau)\right) \tanh r_{k}(\tau)}{1-\exp \left(-2 i \phi_{k}(\tau)\right) \tanh r_{k}(\tau)}\right|\right)^{2}+\left(\tanh ^{-1}\left(\sin \left(2 \phi_{k}(\tau)\right) \sinh \left(2 r_{k}(\tau)\right)\right)\right)^{2}} .
\end{gathered}
$$

We can also obtain approximate expressions for different limiting conditions. These expressions are discussed below:

(a) Small $r_{k}(\tau)$ and Small $\phi_{k}(\tau)$; For small $r_{k}(\tau)$ and $\phi_{k}(\tau)$, we can use approximating expressions, $\exp \left(-2 i \phi_{k}(\tau)\right) \approx 1, \quad \sin \left(2 \phi_{k}(\tau)\right) \approx 2 \phi_{k}(\tau), \quad \tanh$ $r_{k}(\tau) \approx r_{k}(\tau)$, and $\sinh \left(2 r_{k}(\tau)\right) \approx 2 r_{k}(\tau)$ to get

$$
\begin{gathered}
C_{1}\left(\Omega_{k}, \tau\right) \approx 2\left|r_{k}(\tau)\right|\left(1+2\left|\phi_{k}(\tau)\right|\right), \\
C_{2}\left(\Omega_{k}, \tau\right) \approx \sqrt{2}\left|r_{k}(\tau)\right| \sqrt{1+4\left(\phi_{k}(\tau)\right)^{2}} .
\end{gathered}
$$

(b) Large $r_{k}(\tau)$ and Large $\phi_{k}(\tau)$; For large $r_{k}(\tau)$ and $\phi_{k}(\tau)$, we can use the approximating expression $\exp \left(-2 i \phi_{k}(\tau)\right) \approx 0$, and obtain the approximated circuit complexity form

$$
C_{1}\left(\Omega_{k}, \tau\right) \approx\left|\tanh ^{-1}\left(\sin \left(2 \phi_{k}(\tau)\right) \sinh \left(2 r_{k}(\tau)\right)\right)\right|
$$

$C_{2}\left(\Omega_{k}, \tau\right) \approx \frac{1}{\sqrt{2}}\left(\sin \left(2 \phi_{k}(\tau)\right) \sinh \left(2 r_{k}(\tau)\right)\right)$.

These two cost functions are related by $\left|C_{1}\left(\Omega_{k}, \tau\right)\right| \approx \frac{1}{\sqrt{2}} C_{2}\left(\Omega_{k}, \tau\right)$.

(c) Small $r_{k}(\tau)$ and Large $\phi_{k}(\tau)$; For small $r_{k}(\tau)$ and large $\phi_{k}(\tau)$, we can use the approximating expressions: $\exp \left(-2 i \phi_{k}(\tau)\right) \approx 0, \quad \tanh r_{k} \approx r_{k}(\tau), \quad$ and $\sinh$ $\left(2 r_{k}(\tau)\right) \approx 2 r_{k}(\tau)$. This leads to the approximated circuit complexity of the form

$$
\begin{aligned}
& C_{1}\left(\Omega_{k}, \tau\right) \approx 2\left|r_{k}(\tau) \sin \left(2 \phi_{k}(\tau)\right)\right|, \\
& C_{2}\left(\Omega_{k}, \tau\right) \approx \sqrt{2} r_{k}(\tau) \sin \left(2 \phi_{k}(\tau)\right) .
\end{aligned}
$$


These two cost functions are then related by $\left|C_{2}\left(\Omega_{k}, \tau\right)\right| \approx \frac{1}{\sqrt{2}} C_{1}\left(\Omega_{k}, \tau\right)$.

Also, for an example, let us see the structure of the cost functions for large squeezing parameters $r_{k}$ and $\phi_{k} \rightarrow-\frac{\pi}{2}$ :

$C_{1}\left(\Omega_{k}\right) \approx \sqrt{2} C_{2}\left(\Omega_{k}\right) \approx\left|\ln \left(\frac{1-\tanh r_{k}}{1+\tanh r_{k}}\right)\right| \approx r_{k}$.

\section{ENTANGLEMENT ENTROPY OF TWO MODE SQUEEZED STATES}

In this section we will compute the entanglement entropy for the two-mode squeezed states and compare it to the circuit complexity. Not only are our states are entangled, there is also a strong correlation between the two modes. $\left|\psi_{\mathrm{sq}}\right\rangle_{\vec{k},-\vec{k}}$ is also an eigenstate of the difference number operator $\hat{n}_{k}-\hat{n}_{-k}$ with eigenvalue 0 , where $\hat{n}_{k}=\hat{c}_{\vec{k}}^{\dagger} \hat{c}_{-k}$ and $\hat{n}_{-k}=\hat{c}_{-k}^{\dagger} \hat{c}_{\vec{k}}$. Due to this strong correlation and symmetry between the two modes, the average photon number in each mode is same

$$
\left\langle\hat{n}_{k}\right\rangle=\left\langle\hat{n}_{-k}\right\rangle=\sinh ^{2} r_{k}
$$

The reduced density operators for the individual modes are given by

$$
\begin{array}{r}
\hat{\rho}_{k}=\sum_{n=0}^{\infty} \frac{1}{\left(\cosh r_{k}\right)^{2}}\left(\tanh r_{k}\right)^{2 n}\left\langle n_{k} \mid n_{k}\right\rangle, \\
\hat{\rho}_{-k}=\sum_{n=0}^{\infty} \frac{1}{\left(\cosh r_{-k}\right)^{2}}\left(\tanh r_{-k}\right)^{2 n}\left\langle n_{-k} \mid n_{-k}\right\rangle .
\end{array}
$$

The probability of having $n$ photons in a single mode $k$ or $-k$ is

$$
P_{n}^{(i)}=\frac{\left(\tanh r_{k}\right)^{2 n}}{\left(\cosh r_{k}\right)^{2}}, \quad i=k,-k
$$

Commonly used entanglement entropies are von-Neumann and Rényi entanglment entropies. For a density operator $\hat{\rho}$, von-Neumann entropy is given by

$$
S(\hat{\rho})=-\operatorname{Tr}[\hat{\rho} \ln \hat{\rho}] .
$$

If the density operator $\hat{\rho}$ is pure, then $S\left(\hat{\rho}_{\text {pure }}\right)=0$, while for mixed states $S\left(\hat{\rho}_{\text {mixed }}\right)>0$. It is usually not trivial to calculate the entropy. However, for the basis in which the density operator is diagonal such as in the Schmidt basis, the entropy can be calculated simply from the diagonal elements as

$$
S(\hat{\rho})=-\operatorname{Tr}[\hat{\rho} \ln \hat{\rho}]=-\sum_{k} \rho_{k k} \ln \rho_{k k}
$$

Since our two-mode squeezed state (61) is already in the form of a Schmidt decomposition, and we also have the form of reduced density operators of individual modes $a$ and $b$, we can calculate the von-Neumann entanglement entropy by realizing that the diagonal elements $\rho_{k k}$ is $P_{n}^{(i)}$. Then, the von-Neumann entropy, a measure of degree of entanglement is

$$
\begin{aligned}
S\left(\hat{\rho}_{k}\right) & =-\operatorname{Tr}\left[\hat{\rho}_{k} \ln \hat{\rho}_{k}\right]=S\left(\hat{\rho}_{-k}\right) \\
& =-\sum_{n=0}^{\infty} P_{n} \ln P_{n} \\
& =-\sum_{n=0}^{\infty} \frac{\tanh ^{2 n} r_{k}}{\cosh ^{2} r_{k}} \ln \frac{\tanh ^{2 n} r_{k}}{\cosh ^{2} r_{k}} \\
& =-\sum_{n=0}^{\infty} \frac{\tanh ^{2 n} r_{k}}{\cosh ^{2} r_{k}}\left(\ln \left(\tanh ^{2 n} r_{k}\right)-\ln \left(\cosh ^{2} r_{k}\right)\right) \\
& =\ln \left(\cosh ^{2} r_{k}\right) \cosh ^{2} r_{k}-\ln \left(\sinh ^{2} r_{k}\right) \sinh ^{2} r_{k} .
\end{aligned}
$$

We have plotted the von-Neumann entanglement entropy in Fig. 10. It can be seen that entanglement entropy increases with increasing squeezing parameter $r_{k}$. Note that we did not calculate the entropy corresponding to the squeezed state Eq. (61) because naturally this entropy is going to be zero as it is a pure state. Instead, we have calculated entropy for the reduced density matrix.

We can now generalize von-Neumann entropy to get Rényi entropy for the reduced density operator

$$
\begin{aligned}
S_{\mu} & =\frac{1}{1-\mu} \ln \sum_{n=1}^{d} P_{n} \\
& =\frac{2 \mu \ln \operatorname{coshr}_{k}+\ln \left(1-\tanh ^{2 \mu} r_{k}\right)}{\mu-1},
\end{aligned}
$$

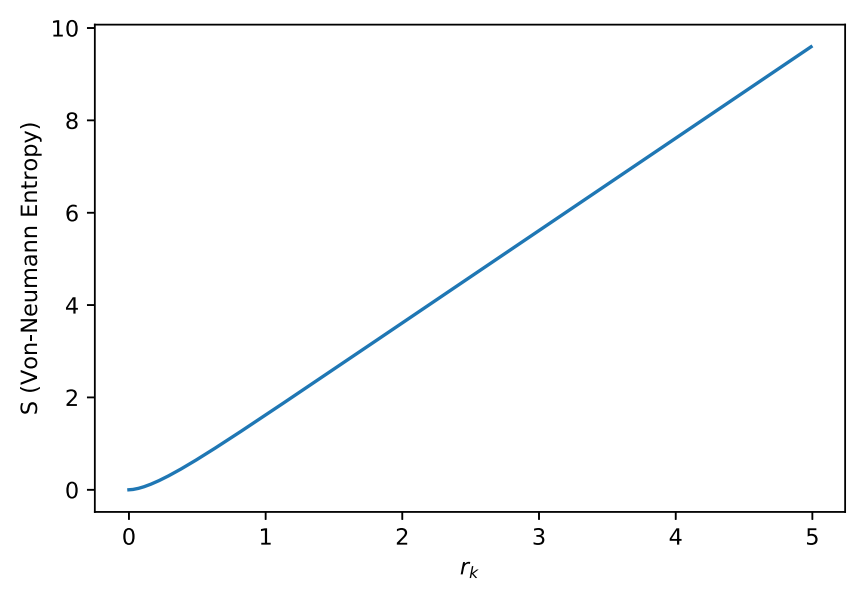

FIG. 10. von-Neumann entanglement entropy $S$ as a function of a squeezing magnitude $r_{k}$. 


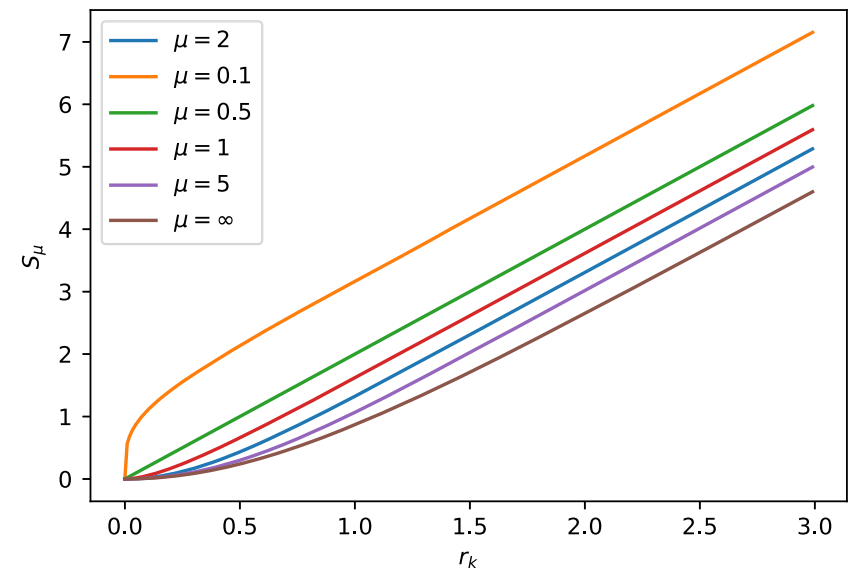

FIG. 11. Rényi-entanglement entropy $S_{\mu}$ as a function of squeezing magnitude $r$. For large $r$, they grow linearly with increasing $r$.

where $\mu \geq 0$ is the Rényi parameter and $d$ is the Schmidt rank of the squeezed state Eq. (61) which is infinity. Again, we can see that the Rényi entropy increases with increasing squeezing parameter $r_{k}$. In Fig. 11 we plotted Rényientanglement entropy for various Rényi parameters, $\mu$.

For very large squeezing parameters we get

$$
S_{\mu}\left(r_{k} \rightarrow \infty\right) \approx \frac{2 \mu r_{k}}{(\mu-1)} .
$$

If we take the limit $\mu \rightarrow 1$, we get the von-Neumann entropy (119). Meanwhile, Rényi-2 entropy is given by $S_{2}\left(r_{k}\right)=\ln \cosh 2 r_{k}$.

One can also calculate the effective temperature of the source by computing the thermal distribution with an average photon number, $\left\langle\hat{n}_{i}\right\rangle=\sinh ^{2} r_{k}$. The average photon number of the thermal field is given by

$$
\left\langle\hat{n}_{i}\right\rangle=\bar{n}=\frac{1}{\exp \left(\hbar \omega / k_{B} T\right)-1} .
$$

Then, one can compute the effective temperature as

$$
\begin{aligned}
T & =\frac{\hbar \omega_{i}}{k_{B}} \ln \left(\frac{\left\langle\hat{n}_{i}\right\rangle}{\left\langle\hat{n}_{i}\right\rangle+1}\right) \\
& =\frac{\hbar \omega_{i}}{k_{B}} \ln \left(\frac{\sinh ^{2} r_{k}}{\sinh ^{2} r_{k}+1}\right) \\
& =\frac{\hbar \omega_{i}}{2 k_{B} \ln \left(\operatorname{coth} r_{k}\right)},
\end{aligned}
$$

where, $\omega_{i}=i / c$ is the frequency of the mode and $i \in(k,-k)$.

\section{A. Quantum circuit complexity vs entanglement}

Now, a comment on comparison of entanglement entropy with circuit complexity is in order. It was very recently shown in [39] that there exists some relationship between entangling power and circuit complexity. Most importantly, if the entanglement entropy grows linearly with time, the geometric-circuit complexity also grows linearly.

Generally, quantum circuit complexity and entanglement are different quantities. However, for small values of circuit cost and entanglement, one can use the entanglement entropy to bound the circuit complexity. The argument presented in [39] is that quantum gates that are close to the identity exhibit little entanglement from the product or entangled states. One of the interesting corollaries presented in [39] is that whenever entanglement entropy grows linearly in time, the circuit complexity also grows linearly. Linear growth of entanglement entropy is a generic feature of several quenched many-body systems.

Our analysis of complexity and entanglement entropy of two-mode squeezed states is in agreement with the result in [39]. Since both entanglement entropy and circuit complexity computed with the covariance matrix method are independent of the squeezing angle $\phi_{k}$, the comparison is clearer than with Nielsen's method of wave functions. The explicit form of circuit complexity with the covariance matrix method is obtained in Eq. (85),

$$
\begin{gathered}
C_{1}(k)=4 r_{k}, \\
C_{2}(k)=2 \sqrt{2} r_{k} .
\end{gathered}
$$

While comparing the form of entanglement entropy Eqs. (119) and (120) with this circuit complexity, we get

$$
C_{1}\left(r_{k}\right)=\sqrt{2} C_{2}\left(r_{k}\right)=4 r_{k} \geq S\left(r_{k}\right) \approx r_{k} .
$$

In Fig. 12, we plotted the comparison between vonNeumann entropy and circuit complexity (computed using the covariance matrix method). The circuit complexities $C_{1}$

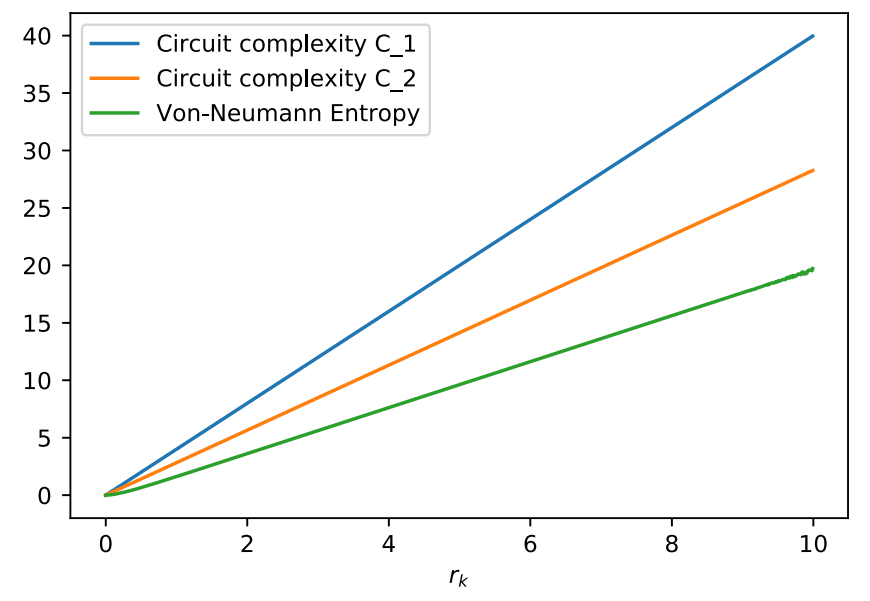

FIG. 12. Comparision of the von-Neumann entanglement entropy with circuit complexity. 
and $C_{2}$ grow linearly just like the entanglement entropy. Up to these distances, circuit complexity is indeed lower bounded by entanglement entropy. This result can have a physical interpretation. Since the entanglement entropy for the two-mode squeezed states increases with increasing $r_{k}$, the entanglement entropy from the vacuum to distant states is large. Therefore, in the context of two-mode squeezed states, with proper circuit complexity cost, entanglement entropy could be used as a measure of complexity.

So far, we have only compared circuit complexity obtained via the covariance approach. A more detailed numerical comparison of circuit complexity via Nielsen's approach with entanglement entropy and temperature will be discussed in the numerical analysis section.

\section{NUMERICAL RESULTS}

In this section we do the numerical analysis of the circuit complexity calculated for the model of "black hole gas". To provide a wholesome and physically relevant discussion, we do the analysis in terms of the scale factor. We begin by solving the evolution equations of the squeezed state parameters given in Eq. (47).

To recast the above differential equations and study the time evolution in terms of a scale factor, a simple change of variable is implemented, which transforms the above equation. This change of variable is sometimes called a field redefinition.

In Fig. 13, we have plotted the evolution of the squeezedstate parameter with respect to the scale factor. The behavior of the squeezed-state parameter $r_{k}$ is crucial for understanding the behavior of the circuit complexity and its evolution with the scale factor. From the behavior of the squeezed state parameters, we see widely different behaviors of the model in $(1+1)$ dimensions i.e., $d=1$ in the plots. The behavior for the higher dimensions however looks to be pretty similar. The squeezing is large and growing at early times; however, after a certain scale, the

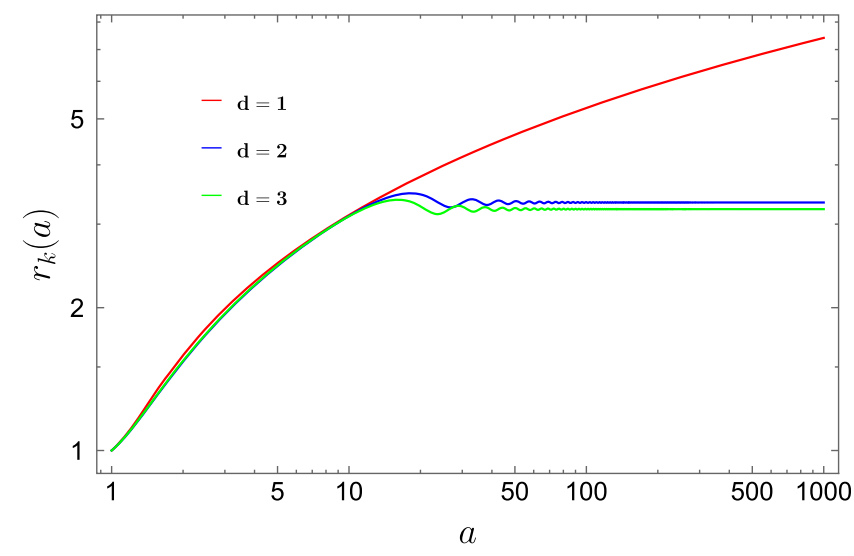

FIG. 13. Behavior of the squeezed-state parameter $r_{k}$ with the scale factor.

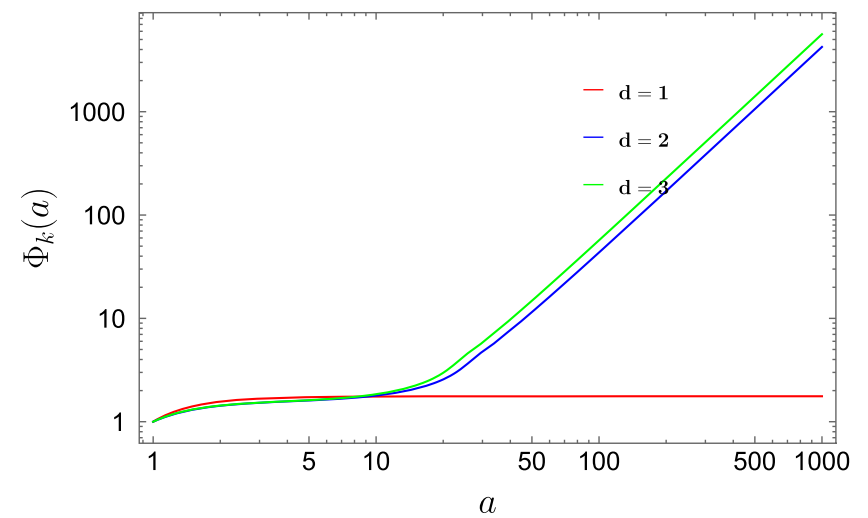

FIG. 14. Behavior of the squeezed state parameter $\phi_{k}$ with the scale factor.

squeezing freezes and saturates at a constant value of squeezing. The increase in the squeezing grows up to a very large scale for spatial dimension $1(d=1$ in the plots) and the freezing of the squeezing effect is not observed even for extremely high scales. This makes the spatial dimension 1 markedly different from the higher spatial dimensions where the freezing effect in the squeezing is explicitly observed.

In Fig. 14, we plotted the squeezing angle $\phi_{k}$ with respect to the scale factor. For the model considered in this paper, we observe that for the spatial dimension 1, the squeezed angle rises for initial scales and is frozen and saturated at intermediate and late scales. However, for higher dimensions the squeezed angle increases at the initial scales but shows a fall after a certain characteristic scale.

In Figs. 15 and 16, we plotted the circuit complexity with respect to the scale factor calculated from the two different cost functionals using both Nielsen's and the covariance approach. Let us make a comparative analysis of complexity obtained from Nielsen's and the covariance approach. The structure of circuit complexity in the covariance approach has a similar pattern as the squeezing parameter $r_{k}$ in Fig. 13 and has almost no feature coming out of the squeezing angle $\phi_{k}$ of Fig. 14. This makes sense as the circuit complexity obtained from the covariance approach is independent of the squeezing angle. Irrespective of the spatial dimension, the circuit complexity $C_{1}$ and $C_{2}$ gradually increase and saturates after some values of $a$.

In contrast to the covariance approach, Nielsen's approach gives a different story of circuit complexity. This is mainly due to the reasoning that the circuit complexity in Nielsen's approach is dependent on both squeezing parameters, $r_{k}$ and $\phi_{k}$, from Figs. 13 and 14. This lets us observe the detail of the evolution of the wave function uniquely. As already pointed out in the previous discussion, the speciality of the spatial dimension 1 can be clearly understood from the complexity plots as well. The initial rise in the complexity measures is observed irrespective of the spatial dimension though the 


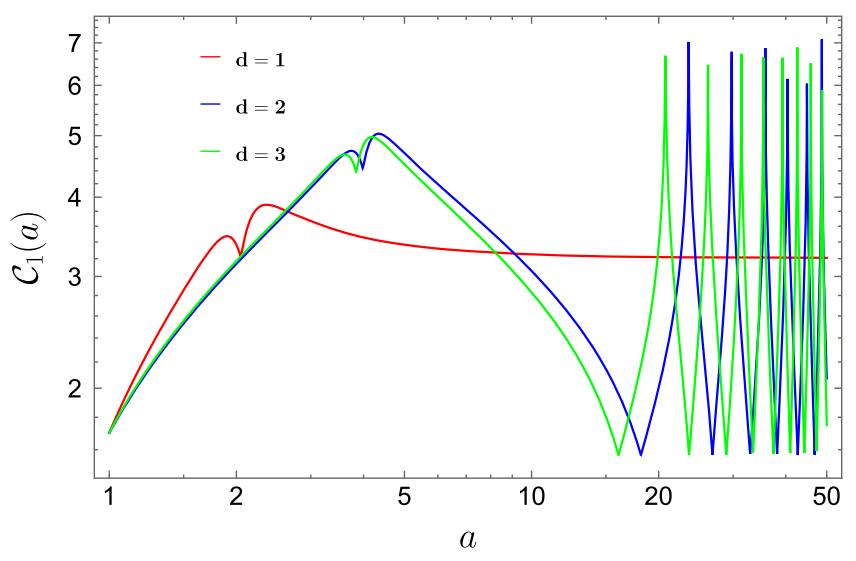

(a)

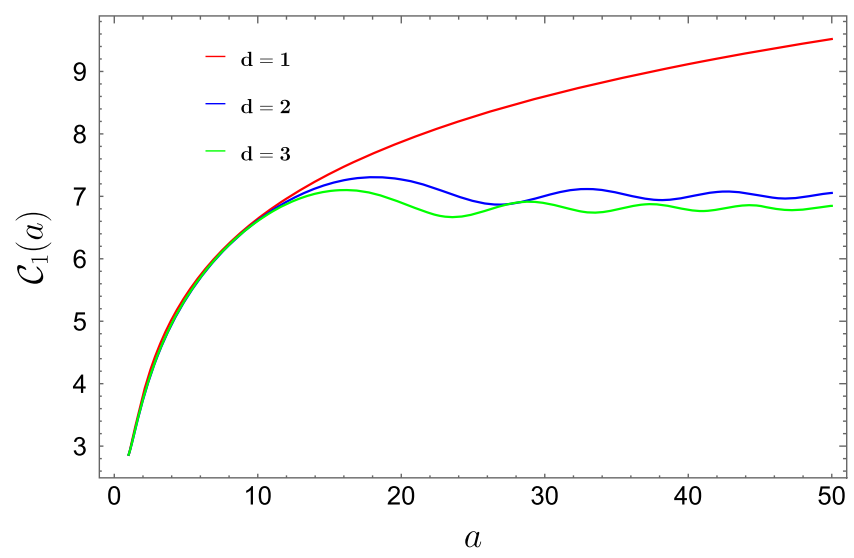

(b)

FIG. 15. Behavior of the linearly weighted circuit complexity $\mathcal{C}_{1}$ with respect to the black hole gas scale factor.

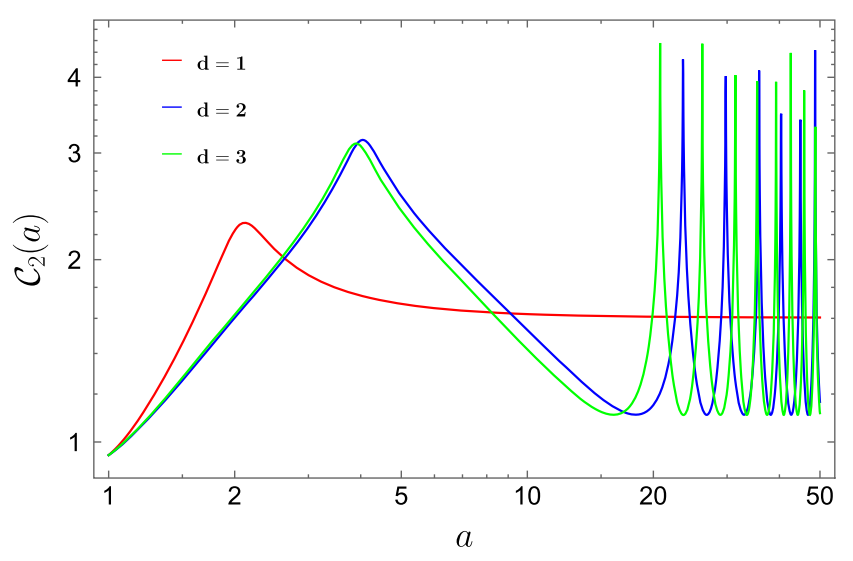

(a)

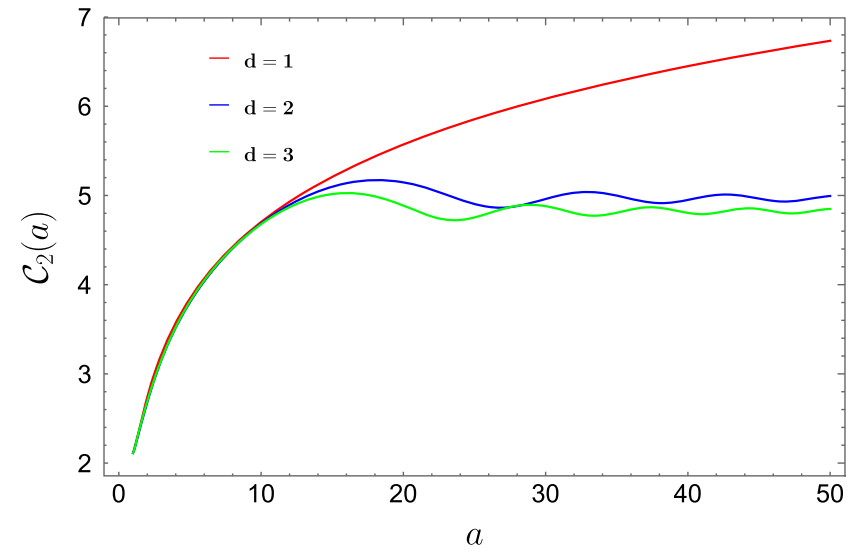

(b)

FIG. 16. Behavior of the geodesically weighted Circuit complexity $\mathcal{C}_{2}$ with respect to the black hole gas scale factor.

scale factor up to which the rise is observed is influenced by the spatial dimension. With the increase in spatial dimension, the rise in the complexities is observed until lower scale factors. After a critical value of the scale factor the complexity measures show a gradual fall in the values. This rate of fall is found to be extremely less for the spatial dimension 1 where even at large value of the scale factor, only a small fall in the value of the complexity is observed. It can be noted that for higher dimension, the complexity measure falls off quickly. The faster a complexity measure falls to a certain minima, the faster it starts to oscillate as seen in the graph. For $d=3$ the oscillation starts early compared to $d=2$. Also, such oscillatory behavior is saturated at higher values of scale factors. The oscillations in Figs. 15 and 16 for higher spatial dimensions could be a hint of the quantum gravity corrections in the very early Universe in terms of vaccum fluctuations of "virtual black holes" of radii R. Such fluctuations could effectively resolve the cosmological constant puzzle. The "vecro component" which describes the part of the wave functional associated to virtual black hole fluctuations that could alter the overall vaccum energy giving us an effective value of cosmological constant $\Lambda=\left(G R^{2}\right)^{-1}$ and hence resolving the issue. The other way to look at the oscillation of complexity is that at the minimum complexity regions, the distance in initial and evolved states are low as one state can be evolved to the next with low number of quantum gates while it is opposite in the maximum complexity regions. So, the structure of the wave function in lower complexity regions are closer to the initial states than the one in high complexity regions.

In Figs. 17-19, we plotted the behavior of entanglement entropy with respect to the scale factor. The two curves in the plots correspond to the two types of entanglement entropy we considered in this paper viz von-Neumann 


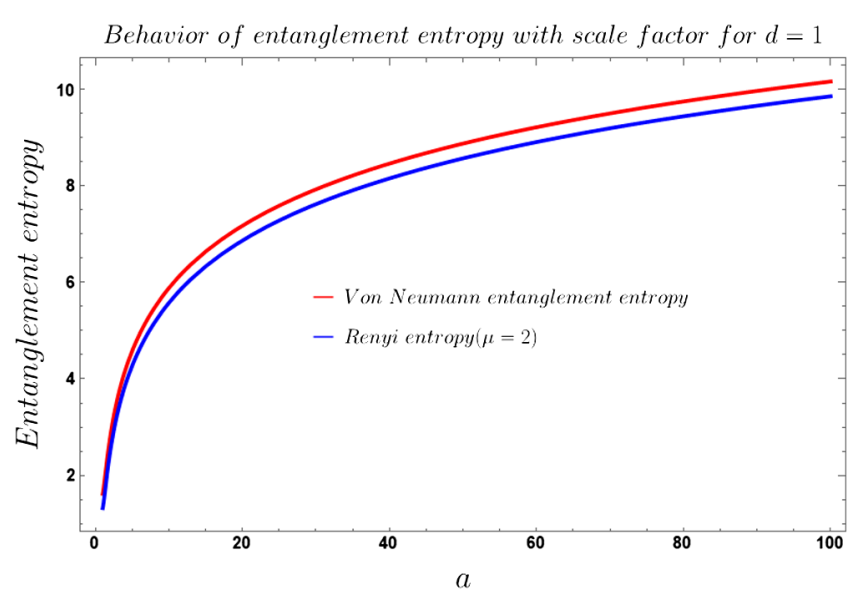

FIG. 17. Behavior of entanglement entropy vs the scale factor for the black hole gas in $d=1$ spatial dimension.

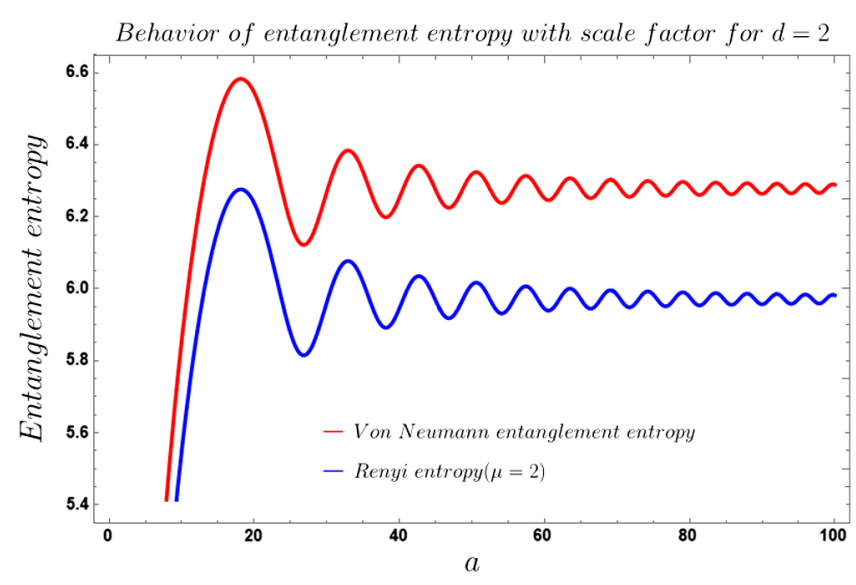

FIG. 18. Behavior of entanglement entropy vs the scale factor for the black hole gas in $d=2$ spatial dimension.

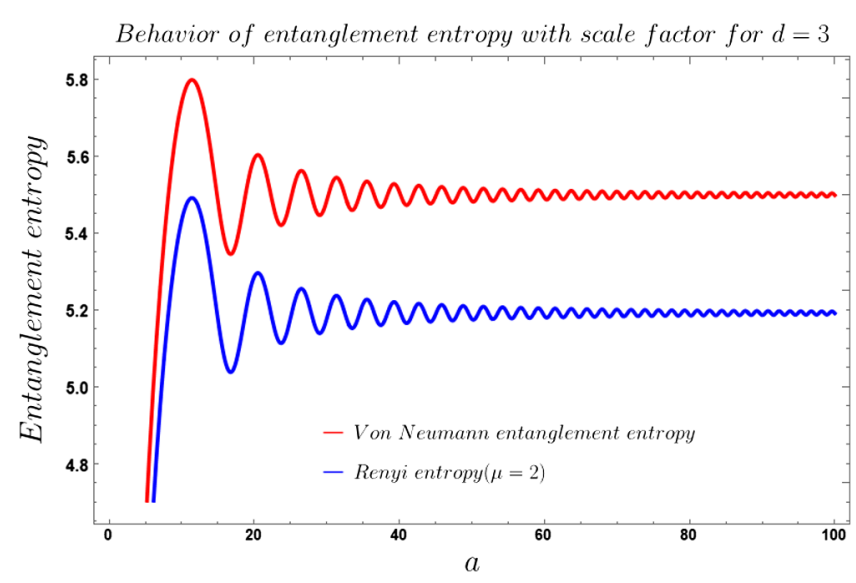

FIG. 19. Behavior of entanglement entropy vs the scale factor for the black hole gas in $d=3$ spatial dimension. entanglement entropy and Rényi entropy. Even though the overall behavior of both forms of entanglement entropy are identical, we still observe a minute difference. It can be seen that the von-Neumann entropy rises faster to a higher value compared to Rényi entropy. This feature is observed for all spatial dimensions. For spatial dimension $d=1$, we observe an increasing behavior of the entropy through the entire range of the scale factor. But for the spatial dimension $d=2,3$, we observe an initial increase in the entropy which then starts to oscillate with its amplitude decaying for higher value of scale factor. It can be noted that with rise in the number of spatial dimension, the rise in entropy decreases and hence saturates at a lower value. We would like to relate the entropy calculated from the squeezed state formalism with the entropy of the black hole gas. One can comment about the entropy of the black hole gas from the entropy calculated using the squeezed state formalism because the information about the black hole gas is itself encoded in the squeezed parameter $r_{k}$. To be more precise, the evolution equations for the squeezed state parameters written in Eq. (47) has been solved using the solution of the scale factor of the black hole gas model as the dynamical variable. Hence, the information about the black hole gas model propagates through the squeezed state parameters to any quantity we calculate. Thus, the entnaglement entropy calculated from the squeezed state parameter is intimately related with the entropy of the black hole gas model.

In Fig. 20, we plotted $d C_{i} / d a$ computed with both Nielsen's and Covariance approach with respect to the von-Neumann entanglement entropy to inspect the validity of the conjectured relation proposed by Susskind between complexity and entanglement entropy. We observe that for the spatial dimension $d=1$, in the initial values of entanglement entropy, the behavior $d C_{i} / d a$ shows an is increasing. However, at the intermediate scales, $d C_{i} / d a$ shows a sharp fall followed by a rise and saturation at large values of entanglement entropy. Thus, we observe a nonlinear relation between $\frac{d C_{i}}{d a}$ and entropy. For low values of entanglement entropy, the difference in amplitude of $d C_{1} / d a$ and $d C_{2} / d a$ is higher in Nielsen's approach than in the covariance approach. This could be because in the covariance approach $C_{1}$ and $C_{2}$ are related by $\mathcal{C}_{1}=\sqrt{2} \mathcal{C}_{2}=4 \sqrt{2} r_{k}$ while in Nielsen's approach $C_{1}$ and $C_{2}$ have a complicated relationship.

In Figs. 21 and 22, we study the behavior of $d C_{i} / d a$ with von-Neumann entanglement entropy for the spatial dimension $d=2$ and $d=3$. We observe an almost identical behavior for the higher spatial dimensions with the behavior shown in spatial dimension 1.

In Figs. 23-25, we have plotted $d C_{i} / d a$ vs Rényi entropy $(\mu=2)$ for different spatial dimensions. It is observed that the overall behavior of $d C_{i} / d a$ with respect to the Rényi entropy is identical to what we observe in the von-Neumann entanglement entropy case. This identical 


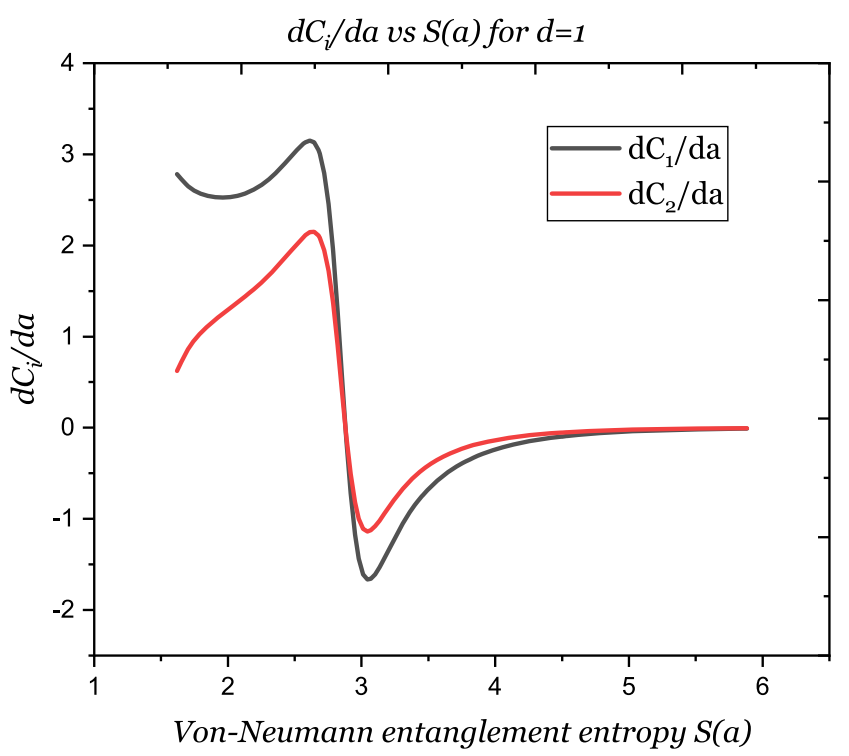

(a)

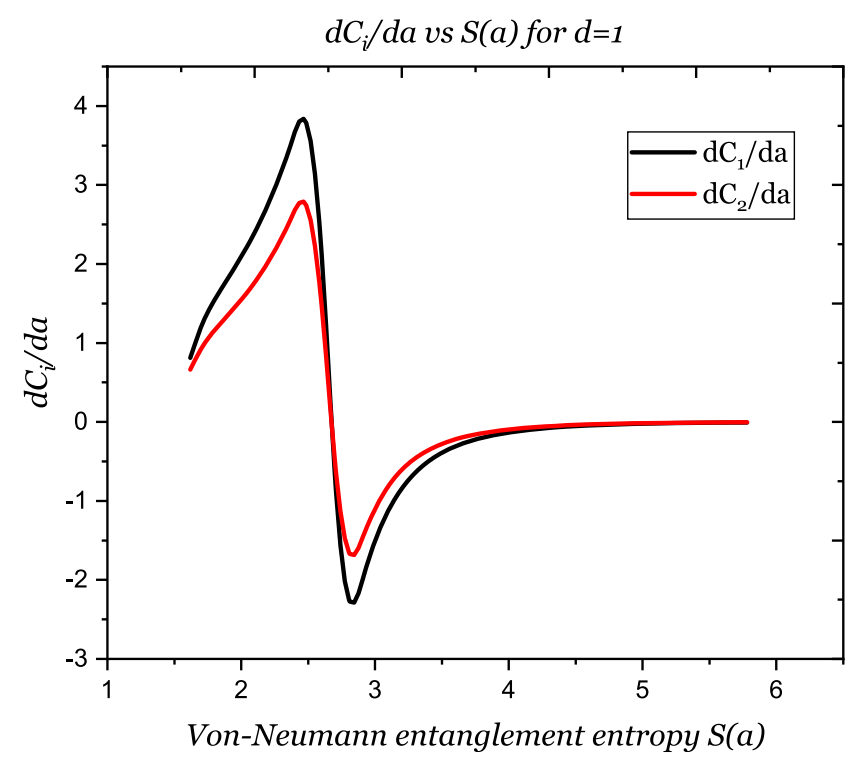

(b)

FIG. 20. Behavior of $d C_{i} / d a$ vs von Neumann entanglement entropy for the black hole gas in $d=1$ spatial dimension.

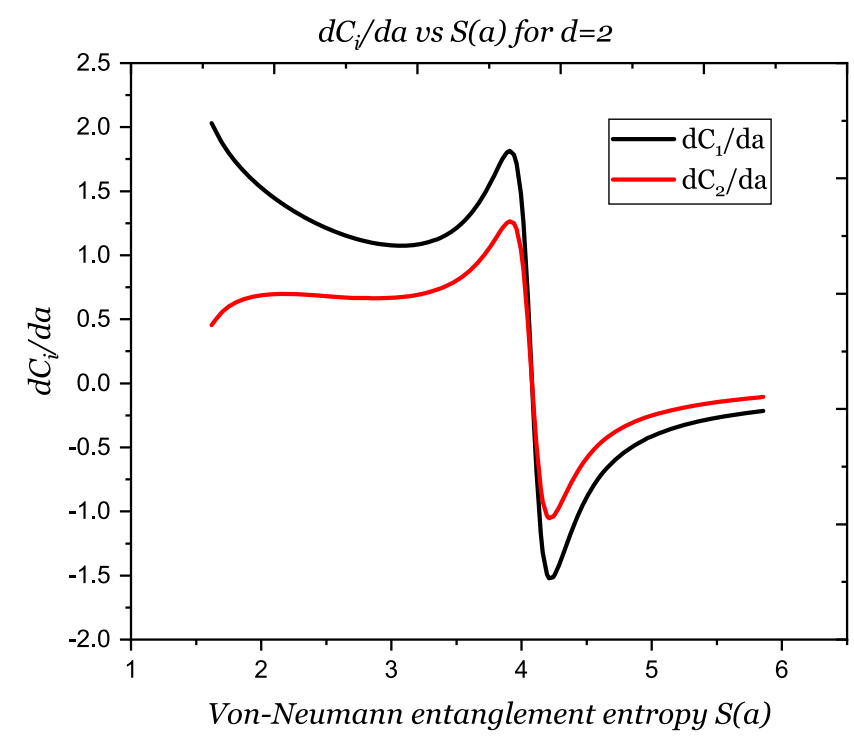

(a)

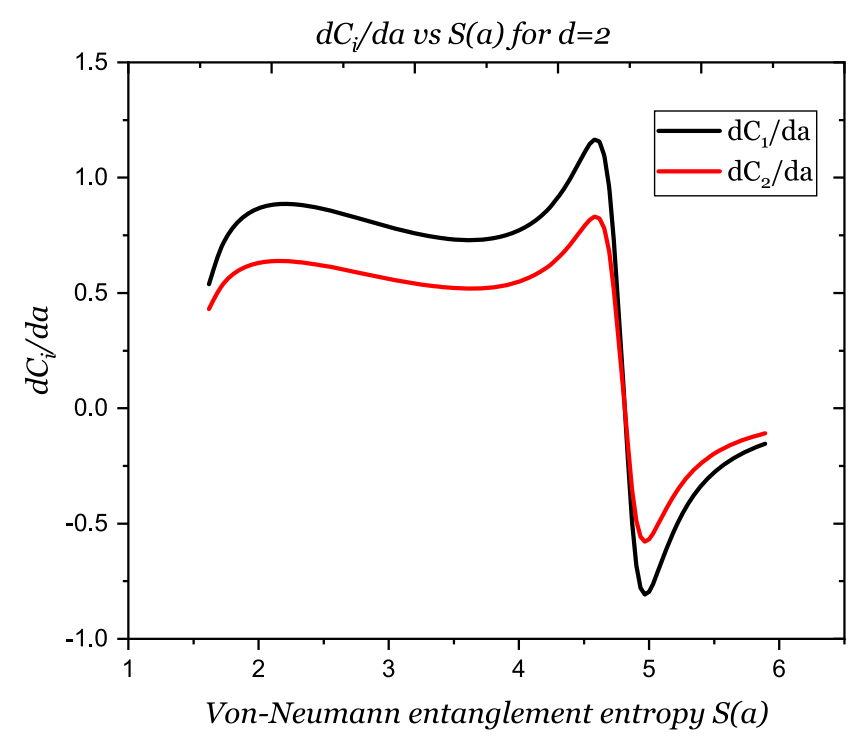

(b)

FIG. 21. Behavior of $d C_{i} / d a$ vs von Neumann entanglement entropy for the black hole gas in $d=2$ spatial dimension.

nature in the behavior of $\frac{d C_{i}}{d a}$ is observed for all spatial dimensions.

In Figs. 26-28 we plotted the behavior of the equilibrium temperature of the black hole gas with respect to $d C_{i} / d a$ and the entanglement entropy for the spatial dimension $d=1,2,3$. The reason we plotted the behavior of the equilibrium temperature with respect to $d C_{i} / d a$ and the entanglement entropy on the same plot was to get an idea of how it behaves with two most important quantities in our analysis i.e., $d C_{i} / d a$ and entanglement entropy. The motivation came from Susskind's conjectured relation where he connected the rate of change of complexity with the entanglement entropy and the equilibrium temperature. However, instead of using $d C_{i} / d t$, we used $d C_{i} / d a$ as we have used the scale factor, which is cosmologically a much more relevant quantity, as the dynamical variable of our 


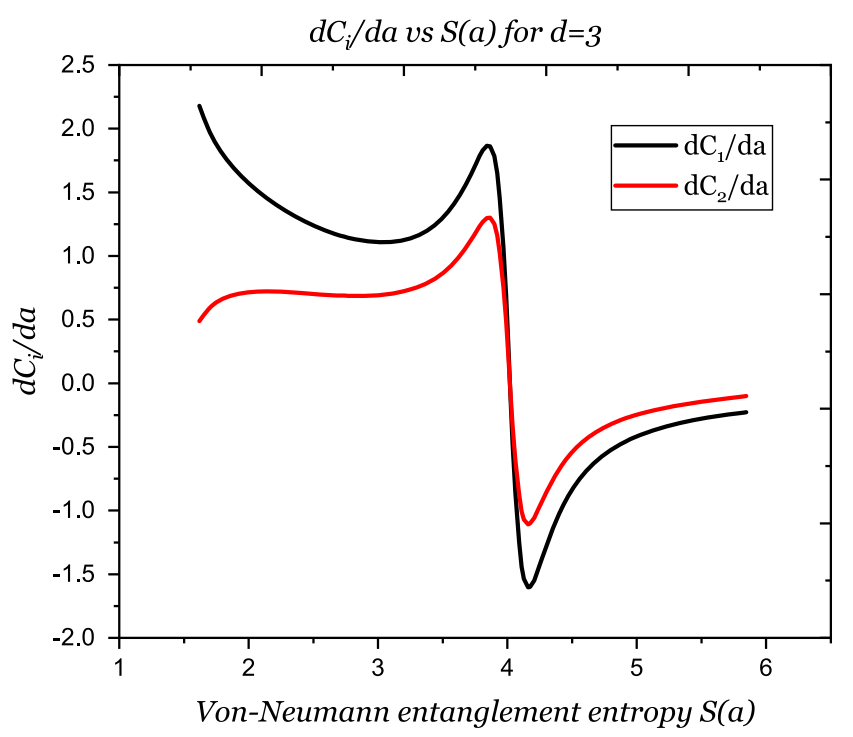

(a)

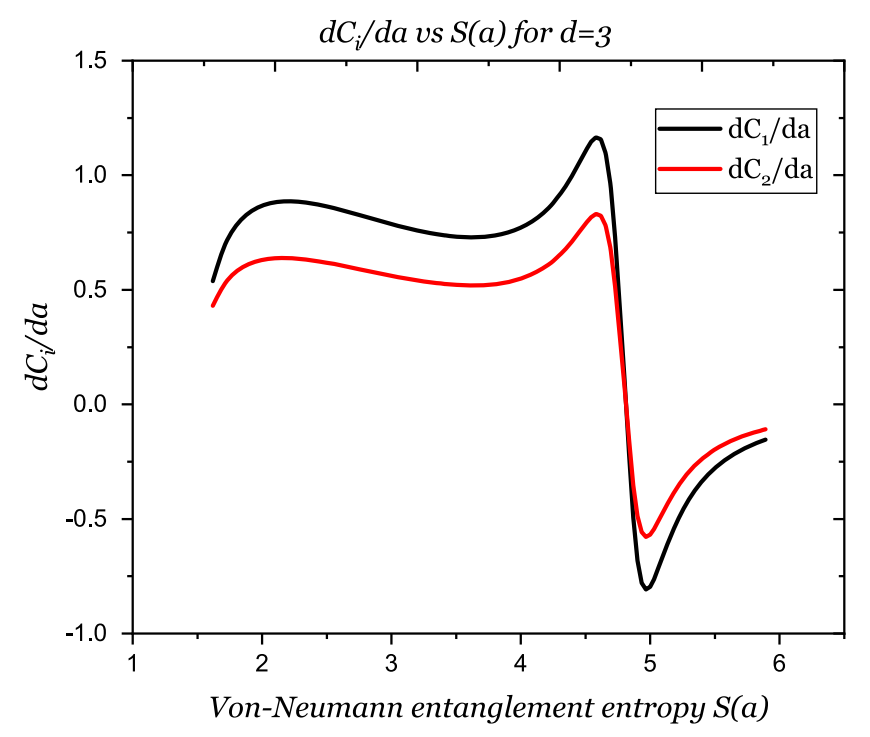

(b)

FIG. 22. Behavior of $d C_{i} / d a$ vs von Neumann entanglement entropy for the black hole gas in $d=3$ spatial dimension.

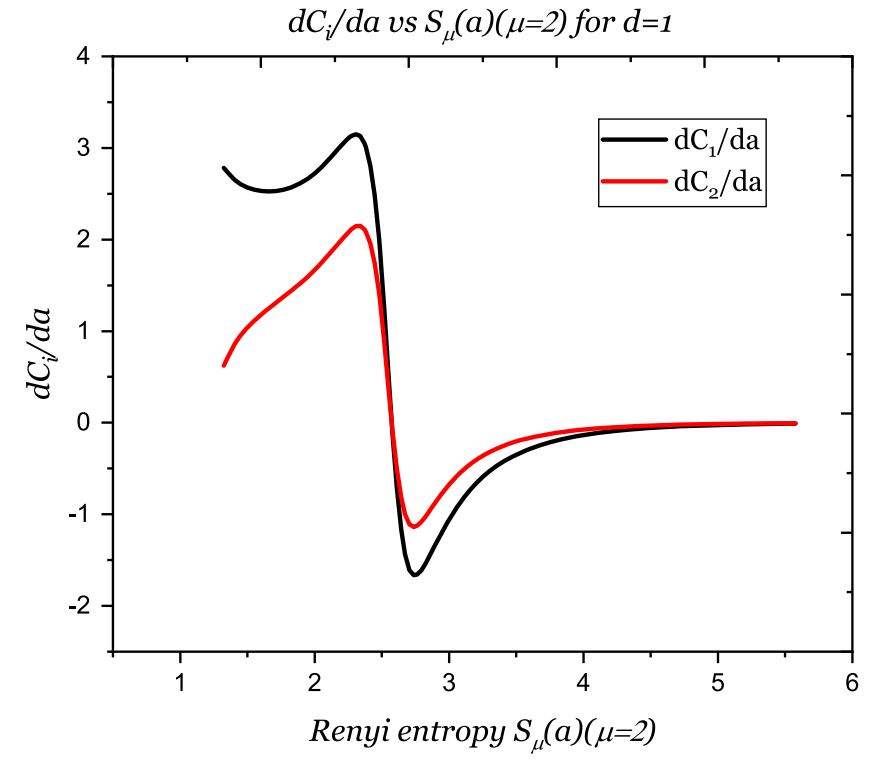

(a)

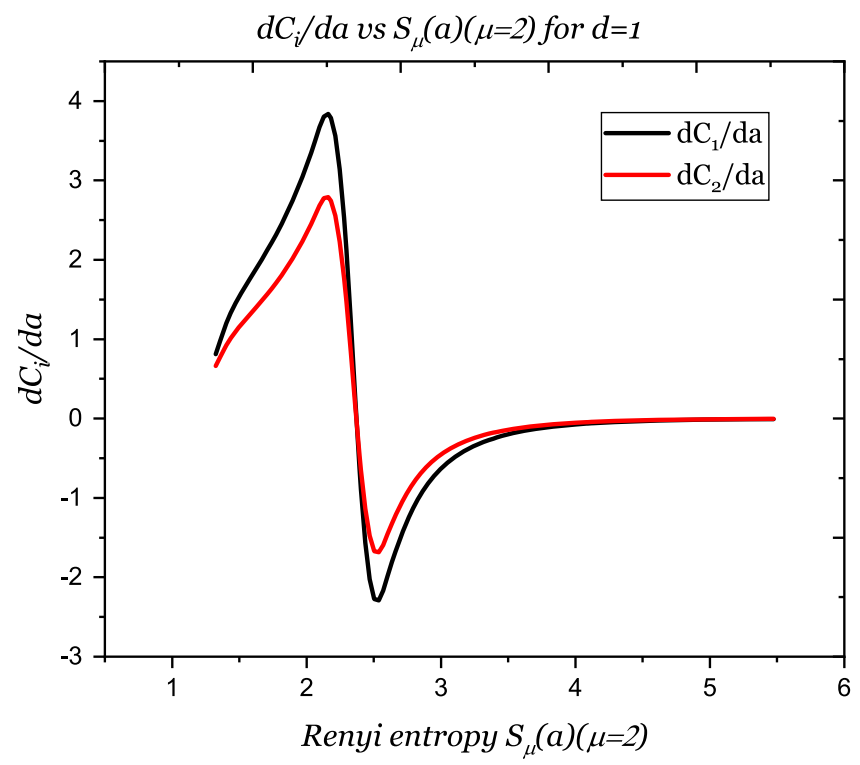

(b)

FIG. 23. Behavior of $d C_{i} / d a$ vs Rényi entropy for the black hole gas in $d=1$ spatial dimension.

analysis. The red curve in the plot shows the behavior of the equilibrium temperature with respect to the entanglement entropy, whereas the black curve shows the behavior with respect to $d C_{i} / d a$. It is clearly evident that irrespective of the spatial dimension, the behavior of the equilibrium temperature shows an increasing. One can approximate the behavior as follows:

$$
T \propto S^{4}
$$

However, it can be seen that the behavior of the equilibrium temperature is overall not identical in nature with $d C_{i} / d a$ for different spatial dimensions and the measure to compute complexity, although some of the features do match. In Nielsen's approach, it can be observed that for negative values of $d C_{i} / d a$, for two values of $d C_{i} / d a$ the black hole gas model attains same value of the equilibrium temperature. For spatial dimension $d=1$, in the intermediate and positive values of $d C_{i} / d a$, the equilibrium temperature is 


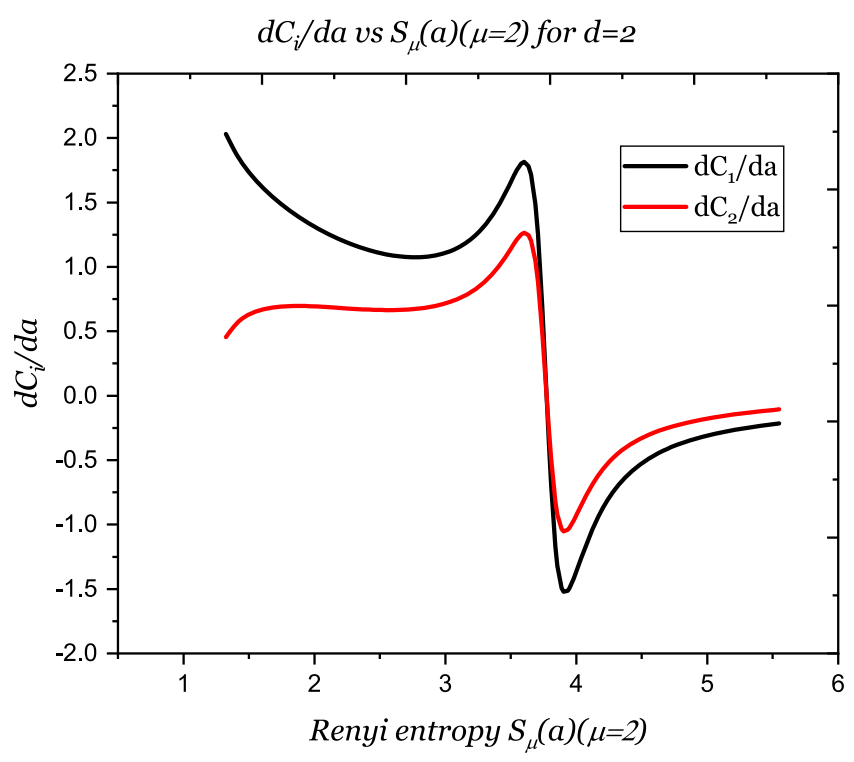

(a)

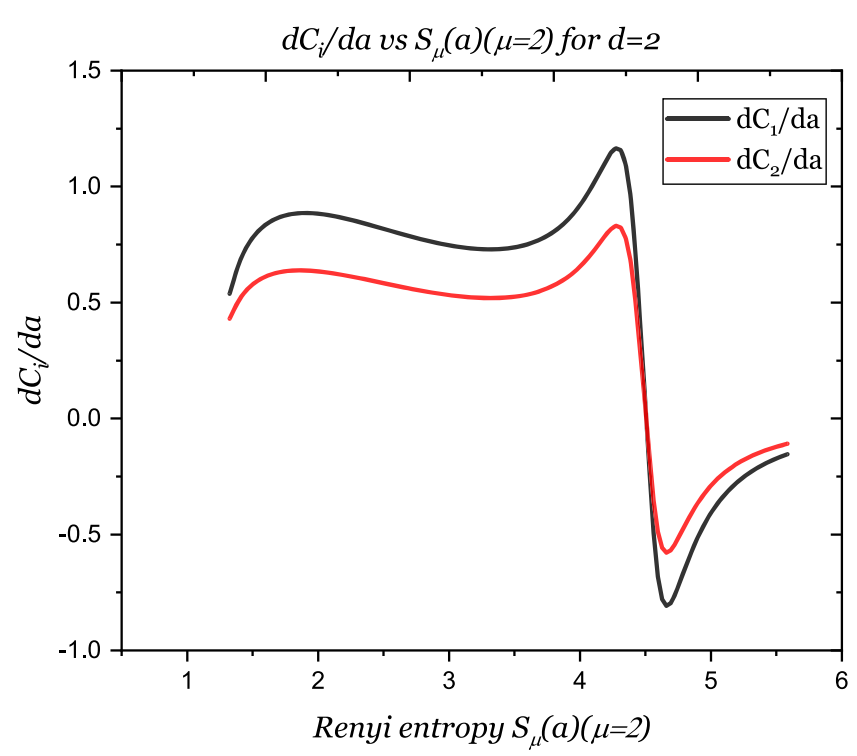

(b)

FIG. 24. Behavior of $d C_{i} / d a$ vs Rényi entropy for the black hole gas in $d=2$ spatial dimension.

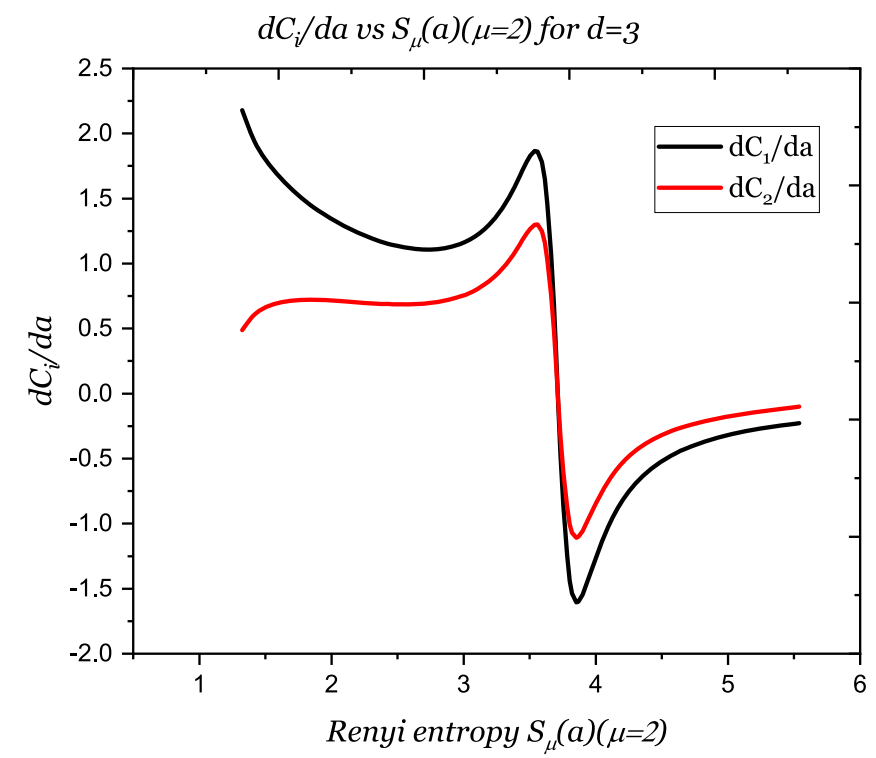

(a)

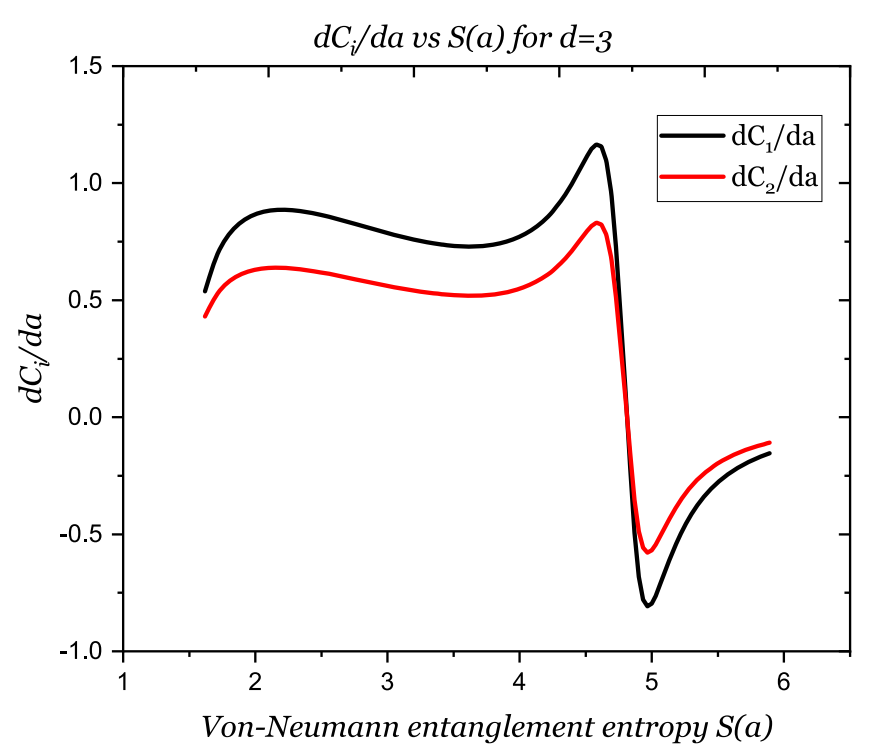

(b)

FIG. 25. Behavior of $d C_{i} / d a$ vs Rényi entropy for the black hole gas in $d=3$ spatial dimension.

almost constant, but for higher spatial dimension the multivalue nature of $d C_{i} / d a$ with the equilibrium temperature returns. However, in the covariance approach in all three spatial dimensions, the behavior is identical.

Thus we see that irrespective of the spatial dimension and the approach of computing complexity in which the black hole model is considered, neither $d C_{i} / d a$ nor entanglement entropy has a linear relationship with the equilibrium temperature.

\section{A. Quantum extremal islands vs black hole gas}

In this portion, we are going to give a comparative analysis of the quantum extremal islands with the black hole gas model from the perspective of circuit complexity.

(a) Circuit complexity calculated from the solution of cosmological islands resembled the page curve in a specific parameter space [36] but for the black hole gas model we observe different behavior of the circuit complexity for different spatial dimension. 


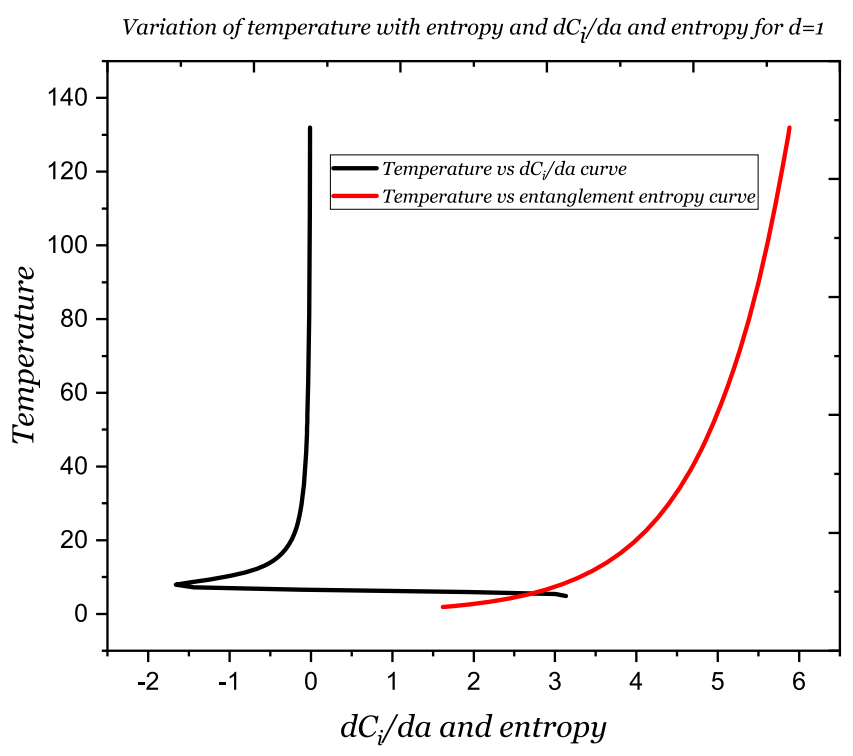

(a)

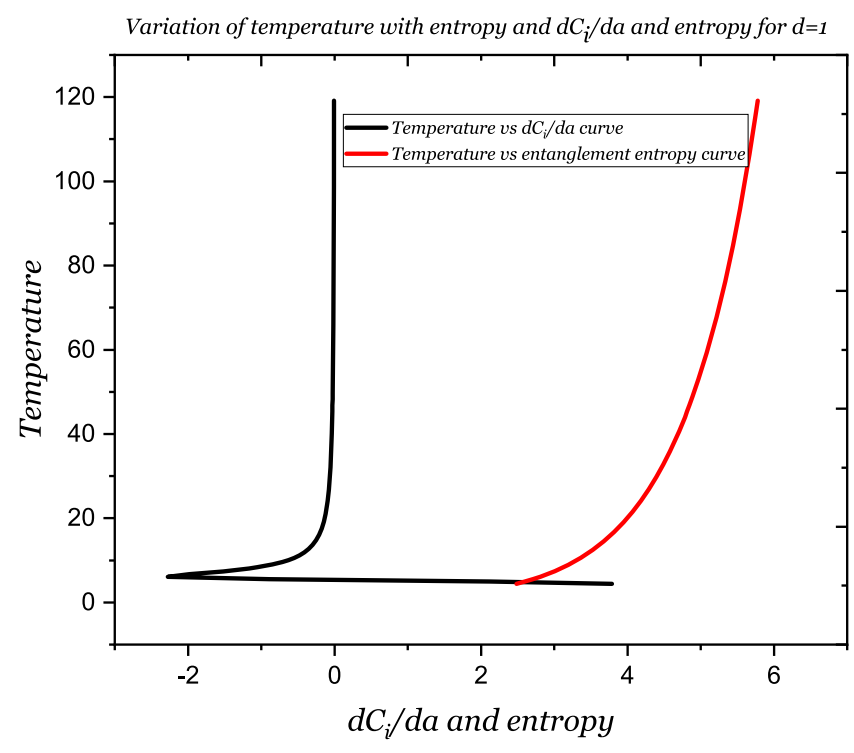

(b)

FIG. 26. Behavior of equilibrium temperature of the black hole gas with respect to $d C_{i} / d a$ and entanglement entropy in $d=1$ spatial dimension.

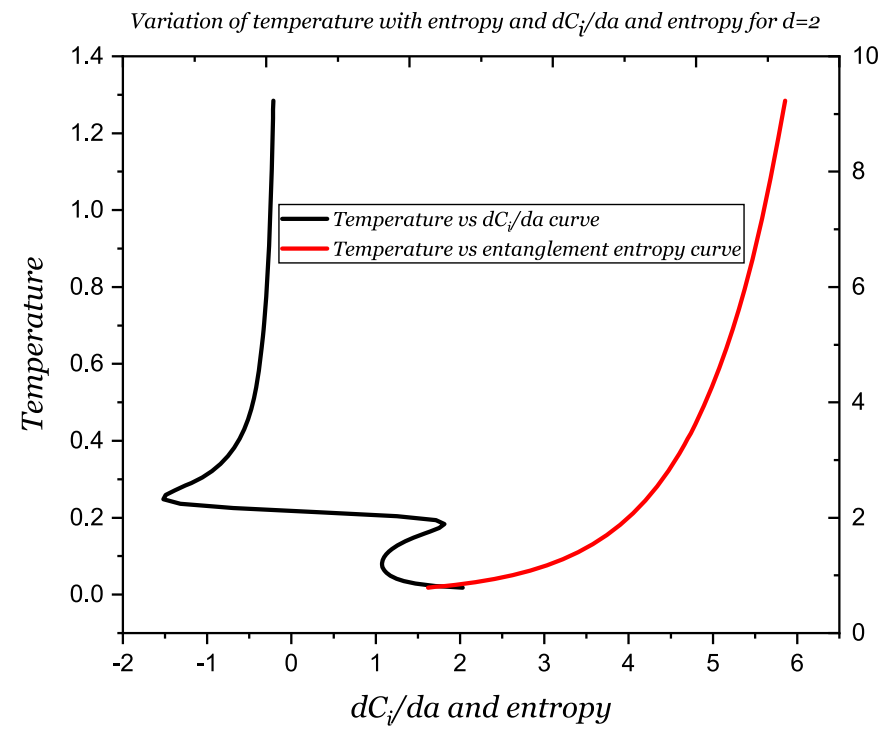

(a)

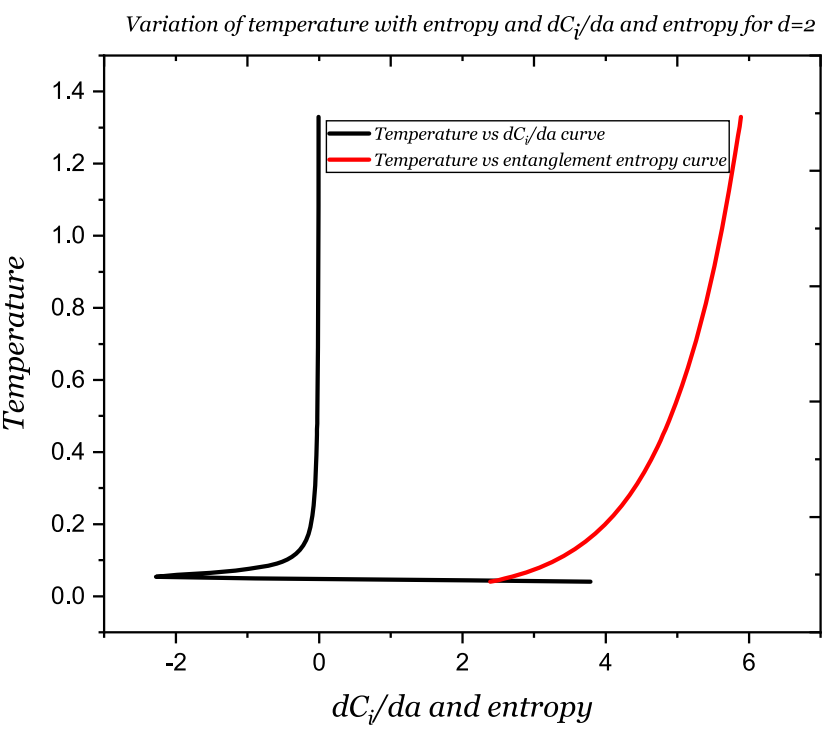

(b)

FIG. 27. Behavior of equilibrium temperature of the black hole gas with respect to $d C_{i} / d a$ and entanglement entropy in $d=2$ spatial dimension.

(b) In another parameter space the behavior of the circuit complexity for the island model showed only a rising behavior which is also different from the one we observe for the black hole gas model.

(c) The entanglement entropy predicted from the circuit complexity in the cosmological island model again resembled the page curve in a particular parameter space and showed a decreasing behavior in another parameter space, whereas for the black hole gas model the entropy showed a increasing behavior for the spatial dimension 1 and 2 and an increasing behavior followed by an oscillation for the spatial dimension 3.

(d) The oscillatory behavior of the circuit complexity at large values of scale factor, which is observed for the higher spatial dimensions for the black hole gas model is absent in the cosmological island model, even when probed to very high scales. 


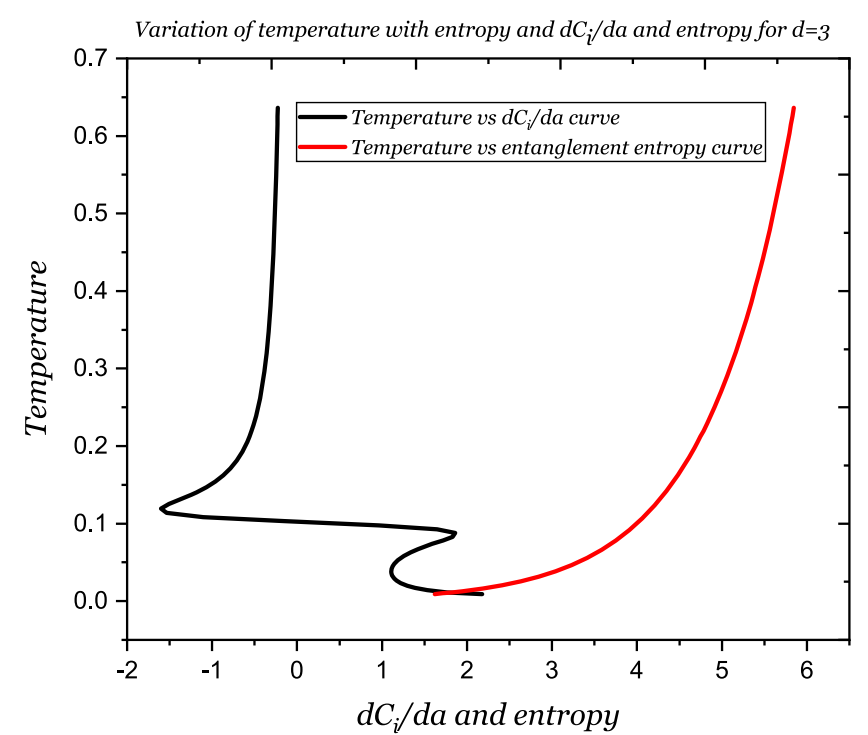

(a)

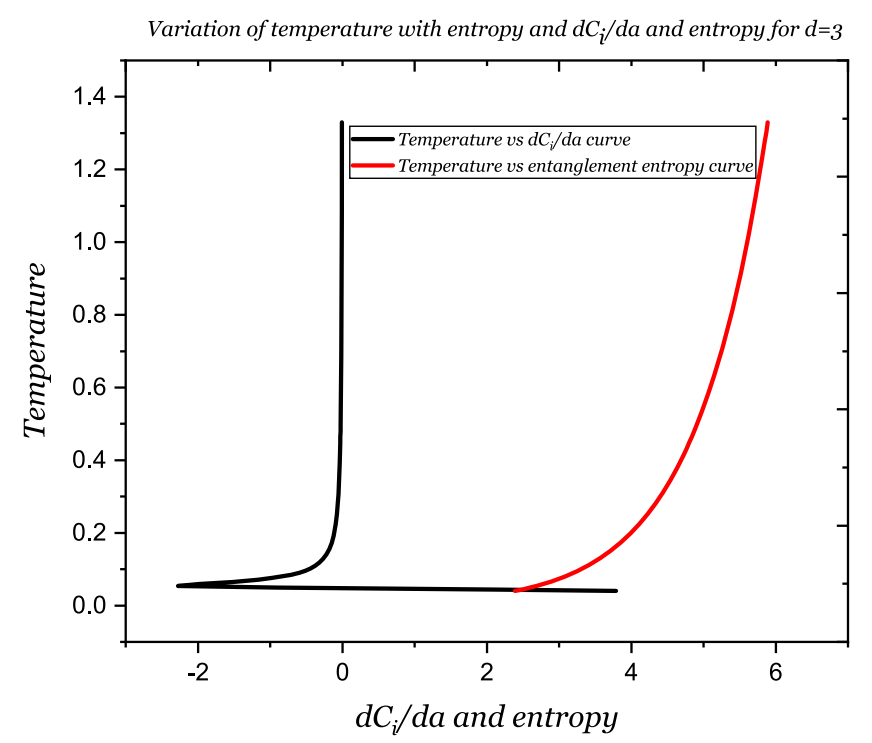

(b)

FIG. 28. Behavior of equilibrium temperature of the black hole gas with respect to $d C_{i} / d a$ and entanglement entropy in $d=3$ spatial dimension.

\section{B. Comparative analysis of circuit complexity from Nielsen's method and the covariance matrix method}

\begin{tabular}{|c|c|c|}
\hline Parameters & Covariance approach & Nielsen's approach \\
\hline $\begin{array}{l}\text { Dependence on squeezing } \\
\text { angle } \phi_{k}\end{array}$ & Does not depend on $\phi_{k}$ & Depends on $\phi_{k}$ \\
\hline $\begin{array}{l}\text { Dependence on squeezing } \\
\text { parameter } r_{k}\end{array}$ & $\begin{array}{l}\text { Always linearly dependent onsqueezing } \\
\text { parameter } r_{k}\end{array}$ & $\begin{array}{l}\text { May have nonlinear dependence on } \\
\text { squeezing parameter } r_{k}\end{array}$ \\
\hline $\begin{array}{l}\text { Sensitivity to details of wave } \\
\text { function }\end{array}$ & $\begin{array}{l}\text { Since it is independent of } \phi_{k} \text {, it is not so sensitive } \\
\text { to the details of the wave function }\end{array}$ & $\begin{array}{l}\text { Since it depends on both } r_{k} \text { and } \phi_{k} \text { it is } \\
\text { sensitive to the details of the wave function. }\end{array}$ \\
\hline Limiting conditions & Only one condition exists $\mathcal{C}_{1}=\sqrt{2} \mathcal{C}_{2}=4 \sqrt{2} r_{k}$ & $\mathcal{C}_{1}$ and $\mathcal{C}_{2}$ are vastly different \\
\hline $\begin{array}{l}\text { Structure of Circuit } \\
\text { complexity in Black Hole } \\
\text { Gas model }\end{array}$ & $\begin{array}{l}\text { For all spatial dimensions it grows until certain } a \\
\text { then, it saturates }\end{array}$ & $\begin{array}{l}\text { Depending on the spatial dimension, it can } \\
\text { oscillate }\end{array}$ \\
\hline $\begin{array}{l}\text { Comparison with genral form } \\
\text { of entanglement entropy }\end{array}$ & $\begin{array}{l}\text { Easier to compare with entanglement entropy as } \\
\text { both are independent of } \phi_{k}\end{array}$ & $\begin{array}{l}\text { Due to dependence on } \phi_{k} \text {, it is difficult to } \\
\text { compare with entanglement entropy. }\end{array}$ \\
\hline $\begin{array}{l}\text { Entanglement entropy and } \\
\text { Circuit Complexity in Black } \\
\text { hole Gas Model }\end{array}$ & $\begin{array}{l}\text { Complexity has the same growth pattern as } \\
\text { entanglement entropy in all three spatial } \\
\text { dimensions which is expected. }\end{array}$ & $\begin{array}{l}\text { It is not trivial to compare complexity with } \\
\text { entanglement entropy. So, one has to do } \\
\text { case by case analysis. }\end{array}$ \\
\hline $\begin{array}{l}d C_{i} / d a \text { and Entanglement } \\
\text { entropy }(S) \text { in Black hole } \\
\text { Gas Model }\end{array}$ & $\begin{array}{l}\text { The difference in amplitude of } d C_{1} / d a \text { and } \\
d C_{2} / d a \text { is lower. }\end{array}$ & $\begin{array}{l}\text { The difference in amplitude of } d C_{1} / d a \text { and } \\
d C_{2} / d a \text { is higher. }\end{array}$ \\
\hline $\begin{array}{l}d C_{i} / d a, \text { Temperature and } \\
\text { entropy }(S) \text { in Black hole } \\
\text { Gas Model }\end{array}$ & $\begin{array}{l}\text { The behavior of Temperature with } d C_{i} / d a \text { and } \\
\text { entropy is different in three different spatial } \\
\text { dimensions. }\end{array}$ & $\begin{array}{l}\text { The behavior of temperature with } d C_{i} / d a \text { and } \\
\text { entropy is same in three different spatial } \\
\text { dimensions. }\end{array}$ \\
\hline
\end{tabular}




\section{CONCLUSIONS}

Through analysis of the black hole gas model from the perspective of circuit complexity and entanglement entropy using the language of the squeezed state formalism we arrive at the following conclusions:

(a) The circuit complexity computed from Nielsen's wave function approach provides a much better understanding than that computed from the covariance matrix method as it depends on both the squeezing angle and the squeezing parameter and hence can be related to the entanglement entropy.

(b) The behavior of the circuit complexity for the spatial dimension $d=1$ is significantly different from higher spatial dimensions. Whereas complexity saturates or changes significantly slowly at large scale factors for $d=1$, it falls off rapidly and has an oscillatory behavior for higher spatial dimensions.

(c) The behavior of the entanglement entropy with respect to the scale factor for different spatial dimension shows different features. For $d=1$, it is just an increasing function whereas for $d=2$ and 3 , we observe an oscillatory behavior with the frequency of oscillation increasing with the increase in spatial dimension.

(d) We observe that for no spatial dimensions the quantity $d C_{i} / d a$ varies linearly with the von-Neumann entanglement entropy or Rényi entropy.

(e) For different spatial dimensions, the behavior of the equilibrium temperature with $d C_{i} / d a$ is peculiar and it is not possible to predict an approximate relation; one has to study different ranges of $d C_{i} / d a$ separately to understand the behavior of the equilibrium temperature.

(f) For different spatial dimensions, from the behavior of equilibrium temperature with entanglement entropy, it can be understood that the relation between entanglement entropy and equilibrium temperature is not linear but goes as

$$
T \propto S^{4}
$$

(g) From the comparative analysis of the black hole gas model with that of the cosmological islands from the perspective of circuit complexity, we can conclude that circuit complexity can be used as a useful tool to discover the underlying features of a model which are otherwise difficult to analyze.

The future prospects of the work can be written:

(a) Circuit complexity has been studied for thermofield double states [20]. The process of thermalization can also be realized by a process known as quantum quench, where the states are expressed as the generalized Calabrese Cardy form. Hence one can explore the thermalization phenomenon using circuit complexity.

(b) People have studied circuit complexity as a deformation in the Euclidean path integral for conformal field theory (CFT's). This is mainly known as path integral optimization [19]. However, these deformations appear in the context of cosmological perturbation theory as well and one can try to extend this circuit complexity using path integral optimization in de Sitter space.

\section{ACKNOWLEDGMENTS}

The research fellowship of S. C. is supported by the J. C. Bose National Fellowship of Sudhakar Panda. S. C. also would line to thank the School of Physical Sciences, National Institute for Science Education and Research (NISER), Bhubaneswar for providing the work friendly environment. S. C. would like to sincerely thank Professor Samir D. Mathur from Ohio State University, USA for various helpful discussions and suggesting the Ref. [40] regarding black hole gas. S. C. also thanks all the members of our newly formed virtual international nonprofit consortium Quantum Structures of the Space-Time and Matter (QASTM) for elaborative discussions. Kiran Adhikari would like to thank TTK, RWTH and JARA, Institute of Quantum Information for fellowships. Satyaki Chowdhury and K. Shirish would like to thank NISER Bhubaneswar and VNIT Nagpur respectively, for providing fellowships. Last but not least, we would like to acknowledge our debt to the people belonging to the various parts of the world for their generous and steady support for research in natural sciences.
[1] S. Chapman, H. Marrochio, and R. C. Myers, Holographic complexity in Vaidya spacetimes. Part I, J. High Energy Phys. 06 (2018) 046.

[2] S. Chapman, H. Marrochio, and R. C. Myers, Holographic complexity in Vaidya spacetimes. Part II, J. High Energy Phys. 06 (2018) 114.

[3] P. A. Cano, R. A. Hennigar, and H. Marrochio, Complexity Growth Rate in Lovelock Gravity, Phys. Rev. Lett. 121, 121602 (2018).
[4] J. L. Barbon and J. Martin-Garcia, Terminal holographic complexity, J. High Energy Phys. 06 (2018) 132.

[5] M. Flory and N. Miekley, Complexity change under conformal transformations in $\mathrm{AdS}_{3} / \mathrm{CFT}_{2}$, J. High Energy Phys. 05 (2019) 003.

[6] S. Chapman, D. Ge, and G. Policastro, Holographic complexity for defects distinguishes action from volume, J. High Energy Phys. 05 (2019) 049. 
[7] C. A. Agón, M. Headrick, and B. Swingle, Subsystem complexity and holography, J. High Energy Phys. 02 (2019) 145.

[8] K. Goto, H. Marrochio, R. C. Myers, L. Queimada, and B. Yoshida, Holographic complexity equals which action?, J. High Energy Phys. 02 (2019) 160.

[9] A. Bernamonti, F. Galli, J. Hernandez, R. C. Myers, S.-M. Ruan, and J. Simón, Aspects of the first law of complexity, J. Phys. A 53, 294002 (2020).

[10] E. Caceres, S. Chapman, J. D. Couch, J. P. Hernandez, R. C. Myers, and S.-M. Ruan, Complexity of mixed states in qft and holography, J. High Energy Phys. 03 (2020) 012.

[11] A. Bernamonti, F. Galli, J. Hernandez, R. C. Myers, S.-M. Ruan, and J. Simón, First Law of Holographic Complexity, Phys. Rev. Lett. 123, 081601 (2019).

[12] K. Goto, H. Marrochio, R. C. Myers, L. Queimada, and B. Yoshida, Holographic complexity equals which action?, J. High Energy Phys. 02 (2019) 160.

[13] M. Guo, J. Hernandez, R. C. Myers, and S.-M. Ruan, Circuit complexity for coherent states, J. High Energy Phys. 10 (2018) 011.

[14] A. Bhattacharyya, A. Shekar, and A. Sinha, Circuit complexity in interacting QFTs and RG flows, J. High Energy Phys. 10 (2018) 140.

[15] R. Khan, C. Krishnan, and S. Sharma, Circuit complexity in fermionic field theory, Phys. Rev. D 98, 126001 (2018).

[16] L. Hackl and R. C. Myers, Circuit complexity for free fermions, J. High Energy Phys. 07 (2018) 139.

[17] D. W. Alves and G. Camilo, Evolution of complexity following a quantum quench in free field theory, J. High Energy Phys. 06 (2018) 029.

[18] H. A. Camargo, P. Caputa, D. Das, M. P. Heller, and R. Jefferson, Complexity as a Novel Probe of Quantum Quenches: Universal Scalings and Purifications, Phys. Rev. Lett. 122, 081601 (2019).

[19] H. A. Camargo, M. P. Heller, R. Jefferson, and J. Knaute, Path Integral Optimization as Circuit Complexity, Phys. Rev. Lett. 123, 011601 (2019).

[20] S. Chapman, J. Eisert, L. Hackl, M. P. Heller, R. Jefferson, H. Marrochio, and R. C. Myers, Complexity and entanglement for thermofield double states, SciPost Phys. 6, 034 (2019).

[21] S. Chapman, M. P. Heller, H. Marrochio, and F. Pastawski, Toward a Definition of Complexity for Quantum Field Theory States, Phys. Rev. Lett. 120, 121602 (2018).

[22] M. Doroudiani, A. Naseh, and R. Pirmoradian, Complexity for charged thermofield double states, J. High Energy Phys. 01 (2020) 120.

[23] S. S. Hashemi, G. Jafari, and A. Naseh, First law of holographic complexity, Phys. Rev. D 102, 106008 (2020).

[24] S. Choudhury, A. Dutta, and D. Ray, Chaos and complexity from quantum neural network: A study with diffusion metric in machine learning, J. High Energy Phys. 04 (2021) 138.

[25] L. Susskind, Three lectures on complexity and black holes, arXiv: 1810.11563.

[26] D. Stanford and L. Susskind, Complexity and shock wave geometries, Phys. Rev. D 90, 126007 (2014).

[27] L. Susskind, Computational complexity and black hole horizons, Fortschr. Phys. 64, 24 (2016); Fortschr. Phys. 64, A44 (2016).
[28] D. A. Roberts, D. Stanford, and L. Susskind, Localized shocks, J. High Energy Phys. 03 (2015) 051.

[29] L. Susskind and Y. Zhao, Switchbacks and the bridge to nowhere, arXiv:1408.2823.

[30] R. Jefferson and R. C. Myers, Circuit complexity in quantum field theory, J. High Energy Phys. 10 (2017) 107.

[31] S. Choudhury, The Cosmological OTOC: Formulating new cosmological micro-canonical correlation functions for random chaotic fluctuations in out-of-equilibrium quantum statistical field theory, Symmetry 12, 1527 (2020).

[32] K. Y. Bhagat, B. Bose, S. Choudhury, S. Chowdhury, R. N. Das, S. G. Dastider, N. Gupta, A. Maji, G. D. Pasquino, and S. Paul, The generalized OTOC from supersymmetric quantum mechanics-study of random fluctuations from eigenstate representation of correlation functions, Symmetry 13, 44 (2020).

[33] S. Choudhury, The cosmological OTOC: A new proposal for quantifying auto-correlated random non-chaotic primordial fluctuations, Symmetry 13, 599 (2021).

[34] K. Hashimoto, K. Murata, and R. Yoshii, Out-of-time-order correlators in quantum mechanics, J. High Energy Phys. 10 (2017) 138.

[35] K. Hashimoto, K.-B. Huh, K.-Y. Kim, and R. Watanabe, Exponential growth of out-of-time-order correlator without chaos: Inverted harmonic oscillator, J. High Energy Phys. 11 (2020) 068.

[36] S. Choudhury, S. Chowdhury, N. Gupta, A. Mishara, S. P. Selvam, S. Panda, G. D. Pasquino, C. Singha, and A. Swain, Circuit complexity from cosmological islands, Symmetry 13, 1301 (2021).

[37] P. Bhargava, S. Choudhury, S. Chowdhury, A. Mishara, S. P. Selvam, S. Panda, and G. D. Pasquino, Quantum aspects of chaos and complexity from bouncing cosmology: A study with two-mode single field squeezed state formalism, arXiv:2009.03893.

[38] S. Choudhury, S. P. Selvam, and K. Shirish, Circuit complexity from supersymmetric quantum field theory with Morse function, arXiv:2101.12582.

[39] J. Eisert, Entangling Power and Quantum Circuit Complexity, Phys. Rev. Lett. 127, 020501 (2021).

[40] S. D. Mathur, Three puzzles in cosmology, Int. J. Mod. Phys. D 29, 2030013 (2020).

[41] P. W. Shor, Algorithms for quantum computation: Discrete logarithms and factoring, in Proceedings 35th Annual Symposium on Foundations of Computer Science (1994), pp. 124-134.

[42] P. W. Shor, Polynomial time algorithms for prime factorization and discrete logarithms on a quantum computer, SIAM J. Sci. Stat. Comput. 26, 1484 (1997).

[43] M. A. Nielsen and I. L. Chuang, Quantum Computation and Quantum Information: 10th Anniversary Edition (Cambridge University Press, Cambridge, England, 2010).

[44] S. Arora and B. Barak, Computational Complexity: A Modern Approach (Cambridge University Press, Cambridge, England, 2007).

[45] C. Moore and S. Mertens, The Nature of Computation (Oxford University Press, New York, 2011).

[46] A. Barenco, C. H. Bennett, R. Cleve, D. P. DiVincenzo, N. Margolus, P. Shor, T. Sleator, J. Smolin, and H. Weinfurter, 
Elementary gates for quantum computation, Phys. Rev. A 52, 3457 (1995).

[47] S. Aaronson, The complexity of quantum states and transformations: From Quantum money to black holes, arXiv:1607.05256.

[48] D. P. DiVincenzo, The physical implementation of quantum computation, Fortschr. Phys. 48, 771 (2000).

[49] M. A. Nielsen, A geometric approach to quantum circuit lower bounds, arXiv:quant-ph/0502070.

[50] M. A. Nielsen, Quantum computation as geometry, Science 311, 1133 (2006).
[51] M. A. N. Mark and R. Dowling, The geometry of quantum computation, arXiv:quant-ph/0701004.

[52] N. Khaneja, S. J. Glaser, and R. Brockett, Sub-riemannian geometry and time optimal control of three spin systems: Quantum gates and coherence transfer, Phys. Rev. A 65, 032301 (2002).

[53] C. Gerry and P. Knight, Introductory Quantum Optics (Cambridge University Press, Cambridge, England, 2004).

[54] T. Ali, A. Bhattacharyya, S. Shajidul Haque, E. H. Kim, and N. Moynihan, Time evolution of complexity: A critique of three methods, J. High Energy Phys. 04 (2019) 087. 